\title{
THE COMPOSED ZERO TRUNCATED LINDLEY-POISSON DISTRIBUTION
}

\author{
Ana Paula Jorge do Espirito Santo ${ }^{1 *}$ and Josmar Mazucheli ${ }^{2}$
}

Received January 23, 2015 / Accepted October 20, 2016

\begin{abstract}
In this paper, a new compounding distribution, named zero truncated Lindley-Poisson distribution is introduced. The probability density function, cumulative distribution function, survival function, failure rate function and quantiles expressions of it are provided. The parameters estimatives were obtained by six methods: maximum likelihood (MLE), ordinary least-squares (OLS), weighted least-squares (WLS), maximum product of spacings (MPS), Cramér-von-Mises (CM) and Anderson-Darling (AD), and intensive simulation studies are conducted to evaluate the performance of parameter estimation. Some generalizations are also proposed. Application in a real data set is given and shows that the composed zero truncated Lindley-Poisson distribution provides better fit than the Lindley distribution and three of its generalizations. The paper is motivated by application in real data set and we hope this model may be able to attract wider applicability in survival and reliability.
\end{abstract}

Keywords: compounding, estimation methods, Lindley distribution, survival analysis, zero truncated Poisson distribution.

\section{INTRODUCTION}

The one parameter Lindley distribution was introduced by Lindley (see, Lindley 1958 and 1965) as a new distribution useful to analyze lifetime data, especially in stress-strength reliability modeling. Suppose that $T_{1}, \ldots, T_{M}$ are independent and identically distributed random variables following the one parameter Lindley distribution with probability density function and distribution function written, respectively, as:

$$
\begin{aligned}
& f_{1}(t \mid \theta)=\frac{\theta^{2}}{(\theta+1)}(1+t) e^{-\theta t} \\
& F_{1}(t \mid \theta)=1-\left(1+\frac{\theta t}{\theta+1}\right) e^{-\theta t}
\end{aligned}
$$

where $t>0$ and $\theta>0$.

\footnotetext{
*Corresponding author.

${ }^{1}$ Universidade Estadual Paulista, DEst, 19060-900 Presidente Prudente, SP, Brasil. E-mail: aplnha_jge@yahoo.com.br

${ }^{2}$ Universidade Estadual de Maringá, DEs, 87020-900 Maringá, PR, Brasil. E-mail: jmazucheli@ gmail.com
} 
For a random variable with the one parameter Lindley distribution, the probability density function, (1), is unimodal for $0<\theta<1$ and decreasing when $\theta>1$ (see Fig. 1-a). The hazard rate function is an increasing function in $t$ and $\theta$ (see Fig. 1-b) and given by:

$$
h_{1}(t \mid \theta)=\frac{\theta^{2}(1+t)}{(1+\theta+\theta t)} .
$$

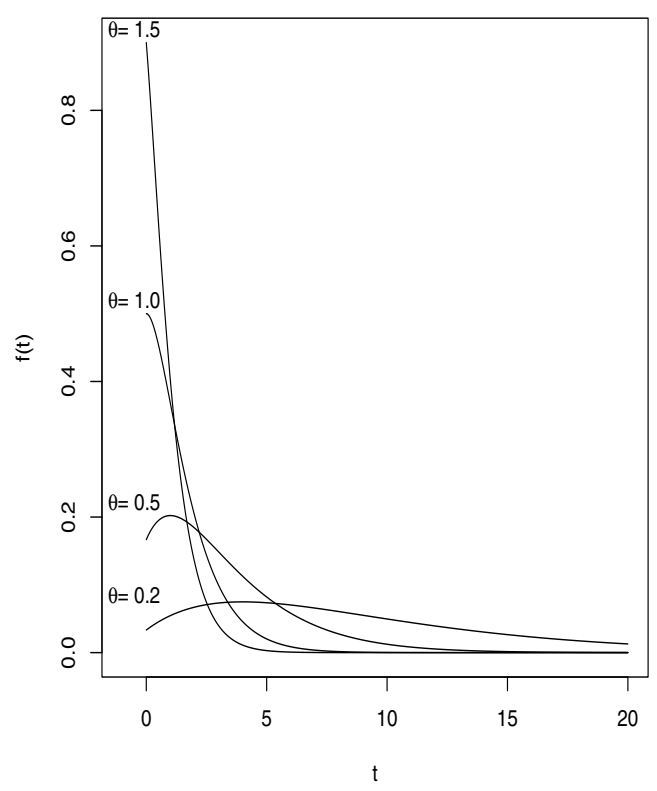

(a)

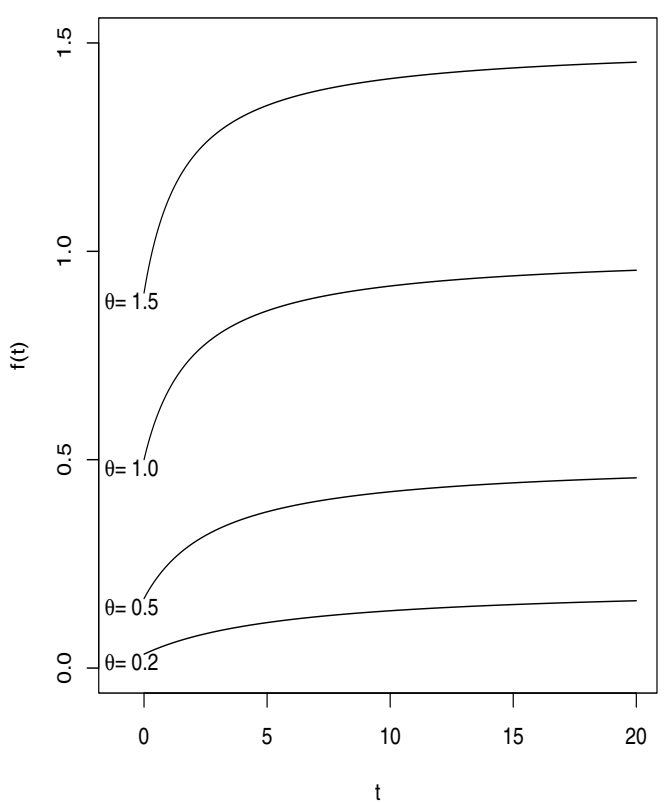

(b)

Figure 1 - Probability density function and hazard rate function behavior for different values of $\theta$.

Ghitany et al. (2008b) studied the properties of the one parameter Lindley distribution under a careful mathematical treatment. They also showed, in a numerical example, that the Lindley distribution is a better model than the Exponential distribution. A generalized Lindley distribution, which includes as special cases the Exponential and Gamma distributions was proposed by Zakerzadeh \& Dolati (2009), and Nadarajah et al. (2011) introduced the exponentiated Lindley distribution. Ghitany \& Al-Mutari (2008) considered a size-biased Poisson-Lindley distribution and Sankaran (1970) proposed the Poisson-Lindley distribution to model count data. Some properties of Poisson-Lindley distribution and its derived distributions were considered in Borah \& Begum (2002) while Borah \& Deka (2001a) considered the Poisson-Lindley and some of its mixture distributions. The zero-truncated Poisson-Lindley distribution and the generalized Poisson-Lindley distribution were considered in Ghitany et al. (2008a) and Mahmoudi \& Zakerzadeh (2010), respectively. A study on the inflated Poisson-Lindley distribution was presented in Borah \& Deka (2001b) and Zamani \& Ismail (2010) considering the Negative BinomialLindley distribution. The weighted and extended Lindley distribution were considered by Ghitany et al. (2011) and Bakouch et al. (2012), respectively. The one parameter Lindley distribution 
in the competing risks scenario was considered in Mazucheli \& Achcar (2011). The exponential Poisson Lindley distribution was presented in Barreto-Souza \& Bakouch (2013). Ghitany et al. (2013) introduced the power Lindley distribution. Ali (2015) investigated various properties of the weighted Lindley distribution which main focus was the Bayesian analysis. A new four-parameter class of generalized Lindley distribution called the beta-generalized Lindley distribution is proposed by Oluyede \& Yang (2015).

Aim to offers more flexible distributions for modeling lifetime data set, in this paper, is proposed an extension of the Lindley distribution. We consider that $T_{j}, j=1, \ldots, M$ is a random sample from the one parameter Lindley distribution and that our variable of interest is defined as:

(i) $Y=\min \left(T_{1}, \ldots, T_{M}\right)$ and $($ ii $) \quad Y=\max \left(T_{1}, \ldots, T_{M}\right)$

representing, respectively, the first and the last failure time of a certain device subject to the presence of an unknown number $M$ of causes of failures. Furthermore, we consider that $M$ has a zero truncated Poisson distribution, $M \sim \operatorname{PoissonTrunc}(\lambda), \lambda>0$, and that $T_{j}$, $j=1, \ldots, M$, and $M$ are independent random variables, leading to the composed zero truncated Lindley-Poisson distribution. The process of composition using the zero truncated Poisson distribution has been fairly used in the literature. In Kuş (2007) was considered the zero truncated Exponential-Poisson distribution in the competing risks scenario. Hemmati et al. (2011) developed the zero truncated Weibull-Poisson distribution. Also in 2011, the same distribution was studied by Ristić \& Nadarajah (2012) and Lu \& Shi (2012). The zero truncated Exponential-Poisson distribution in the complementary risks scenario was introduced by Rezaei \& Tahmasbi (2012).

The paper is organized as follows: in Section 2 the zero truncated Lindley-Poisson distribution is formulated. In Section 3 six estimation methods are presented. A simulation study is introduced in Section 4. The Section 5 brings a real data application. And finally, conclusions are presented in Section 6.

\section{MODEL FORMULATION}

In the theory of competing risks and complementary risks the number of risk factors (or causes) that may lead to the event of interest, is known and denoted as $M$. However, in models of distributions composition is assumed that $M$ is unknown. Therefore, there is a number $M$ of latent risk factors competing to cause the event of interest. In what follows, let us consider the situation where an individual or unit is exposed to $M$ possible causes of death or failure, such that the exact cause is fully known (David \& Moeschberger, 1978). The model for lifetime in the presence of suchcompeting risks structure or complementary risks structure is known as model of composition distributions. If $T_{j}, j=1, \ldots, M$ denote the latent failure times of a individual subject to $M$ risks, which are independent of $M$, what is observed is the time to failure $Y=\min \left(T_{1}, \ldots, T_{M}\right)$. Given $M=m$, under the assumption that the latent failure times $T_{j}$, $j=1, \ldots, M$ are independent and identically distributed random variables with the distribution 
function (2), the probability density function and the cumulative distribution function are written, respectively, as:

$$
\begin{aligned}
& f(y \mid M=m, \theta)=\frac{m \theta^{2}(1+y) e^{-\theta y}}{\theta+1}\left[\left(1+\frac{\theta y}{\theta+1}\right) e^{-\theta y}\right]^{m-1}, \\
& F(y \mid \theta, M=m)=1-\left[\left(1+\frac{\theta y}{\theta+1}\right) e^{-\theta y}\right]^{m} .
\end{aligned}
$$

It is important to note that (4) and (5) are uniquely determined by the distribution function of the minimum, that is, $P(Y \leq y)=1-\left[1-F_{1}(y \mid \theta)\right]^{m}$, (Arnold et al., 2008).

Now, assuming the number of causes of death or failure, $M$, is a zero truncated Poisson random variable with probability mass function given by:

$$
P(M=m)=\frac{\lambda^{m} e^{-\lambda}}{m !\left(1-e^{-\lambda}\right)},
$$

where $m=1,2, \ldots$ and $\lambda>0$, Rezaei \& Tahmasbi (2012).

The marginal probability density function, $f_{\min }(y \mid \theta, \lambda)$, the marginal cumulative distribution function, $F_{\min }(y \mid \theta, \lambda)$, and the marginal hazard rate function, $h_{\min }(y \mid \theta, \lambda)$ of $Y=$ $\min \left(T_{1}, \ldots, T_{M}\right)$ are given, respectively, by:

$$
\begin{aligned}
f_{\min }(y \mid \theta, \lambda) & =\frac{\lambda \theta^{2}(1+y) e^{-\left[\theta y+\lambda\left(1-\left(1+\frac{\theta y}{\theta+1}\right) e^{-\theta y}\right)\right]}}{(\theta+1)\left(1-e^{-\lambda}\right)}, \\
F_{\min }(y \mid \theta, \lambda)= & \frac{1-e^{-\lambda\left[1-\left(1+\frac{\theta y}{\theta+1}\right) e^{-\theta y}\right]}}{1-e^{-\lambda}}, \\
h_{\min }(y \mid \theta, \lambda)= & \frac{\lambda \theta^{2}(1+y) e^{-\left[\theta y+\lambda\left(1-\left(1+\frac{\theta y}{\theta+1}\right) e^{-\theta y}\right)\right]}}{(\theta+1)\left[e^{-\lambda\left[1-\left(1+\frac{\theta y}{\theta+1}\right) e^{-\theta y}\right]}-e^{-\lambda}\right]}
\end{aligned}
$$

where $\theta>0, \lambda>0$ and $y>0$, which defines the zero truncated Lindley-Poisson distribution in the competing risks scenario. Taking the $\lambda=0$ we have the one parameter Lindley distribution as a particular case. Note that $f_{\min }(0 \mid \theta, \lambda)=\frac{\lambda \theta^{2}}{(\theta+1)\left(1-e^{-\lambda}\right)}$ and $f_{\min }(\infty \mid \theta, \lambda)=0$. For all $\theta>0$ and $\lambda>0$, the probability density function, (7), is decreasing or unimodal (see Fig. 2). For values of $\lambda$ close to 1 , the curve resembles the one parameter Lindley distribution, while when $\lambda \longrightarrow 0$ the curve tends to be symmetric.

The hazard rate, (9), is increasing, increasing-decreasing-increasing and decreasing (see Fig. 3). Is easy to see that $h_{\min }(0 \mid \theta, \lambda)=f_{\min }(0 \mid \theta, \lambda)=\frac{\lambda \theta^{2}}{(\theta+1)\left(1-e^{-\lambda}\right)}$ and $h_{\min }(\infty \mid \theta, \lambda)=\theta$.

Now, under the same assumptions and considering a complementary risks scenario, (Basu, 1981), where $Y=\max \left(T_{1}, \ldots, T_{M}\right)$ is observed, the marginal probability density function, 

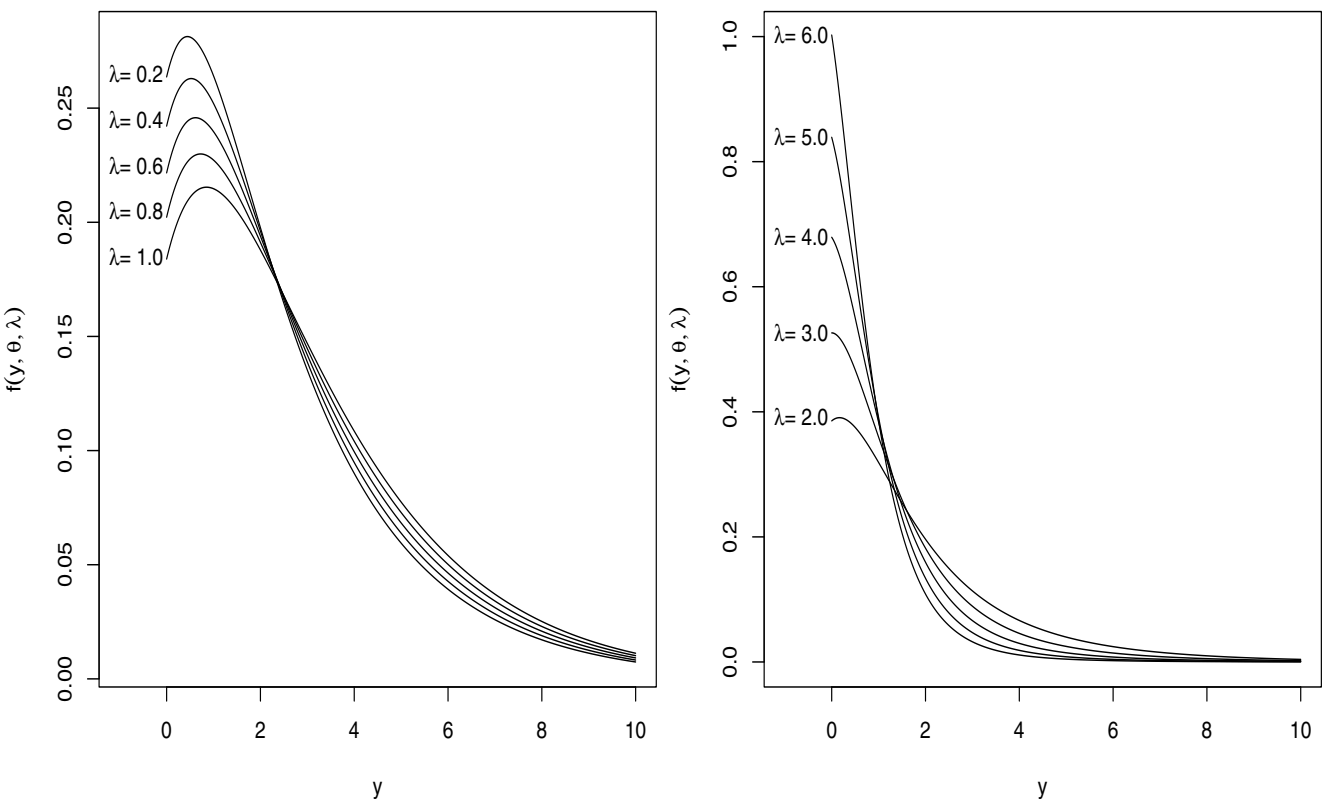

Figure 2 - The zero truncated Lindley-Poisson probability density function for different values of the $\lambda$ and $\theta=0.5$ if $Y=\min \left(T_{1}, \ldots, T_{M}\right)$.
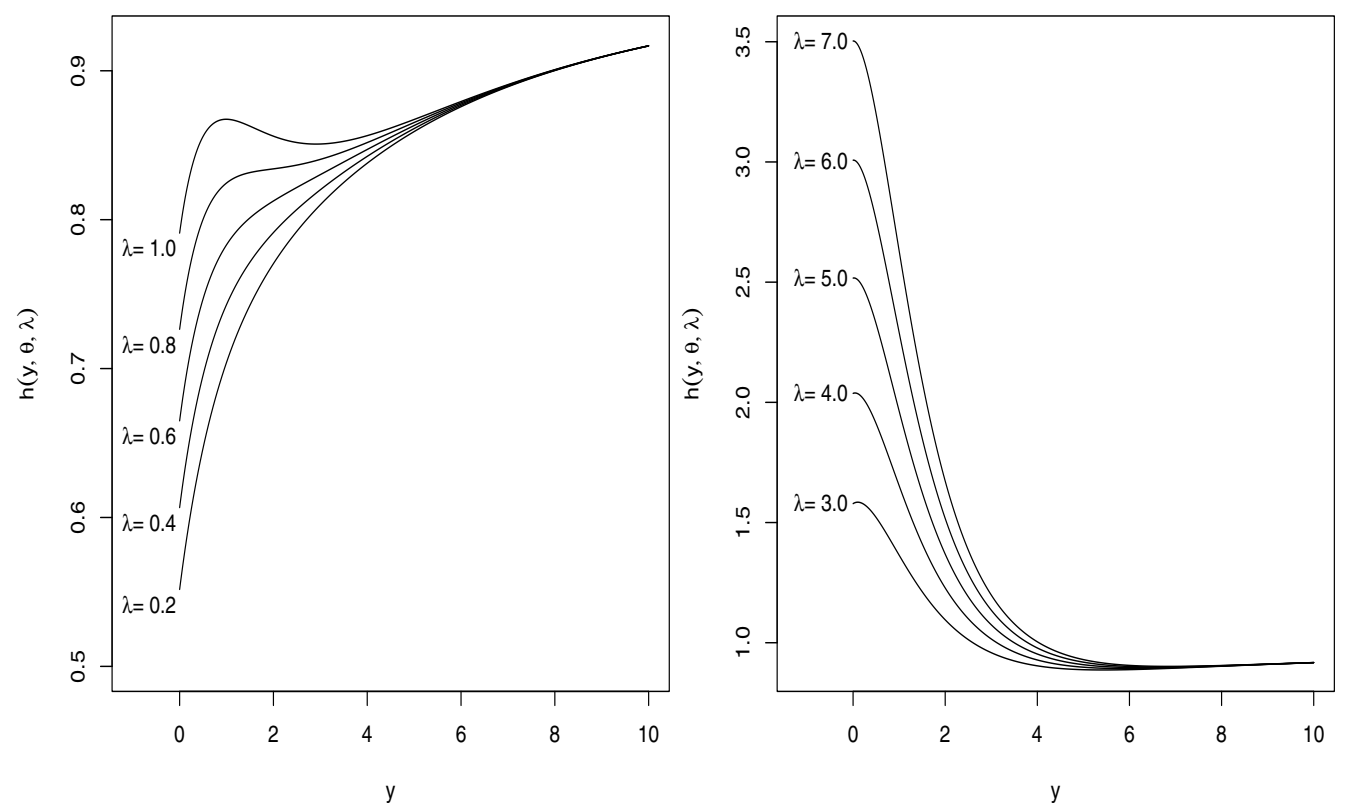

Figure 3 - The zero truncated Lindley-Poisson hazard rate function for different values of the $\lambda$ and $\theta=2.0$ if $Y=\min \left(T_{1}, \ldots, T_{M}\right)$. 

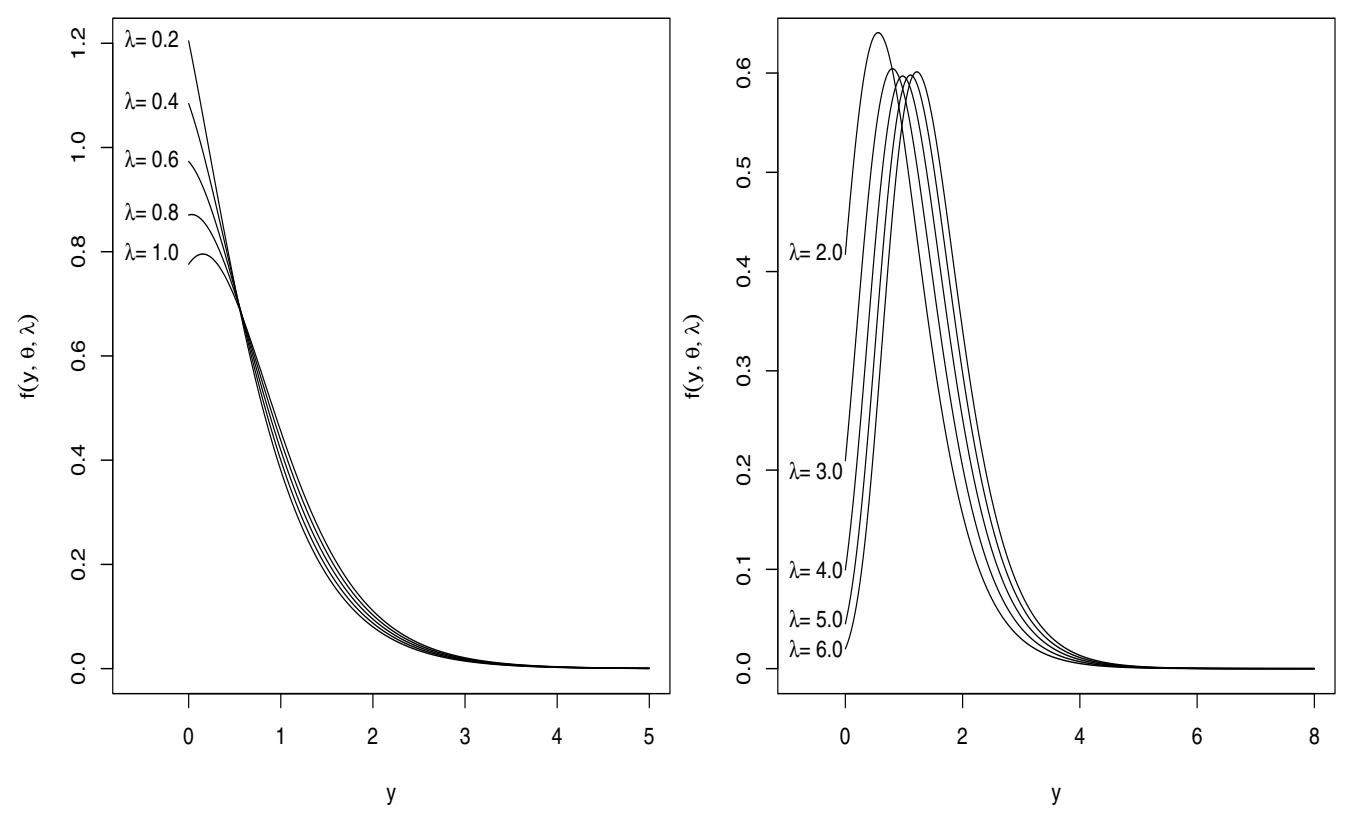

Figure 4 - The zero truncated Lindley-Poisson probability density function for different values of the $\lambda$ and $\theta=2.0$ if $Y=\max \left(T_{1}, \ldots, T_{M}\right)$.

$f_{\max }(y \mid \theta, \lambda)$, the cumulative distribution function, $F_{\max }(y \mid \theta, \lambda)$, and the hazard rate function, $h_{\max }(y \mid \theta, \lambda)$, are given, respectively, by:

$$
\begin{aligned}
f_{\max }(y \mid \theta, \lambda) & =\frac{\lambda \theta^{2}(1+y) e^{-\left[\theta y+\lambda\left(1+\frac{\theta y}{1+\theta}\right) e^{-\theta y}\right]}}{(1+\theta)\left(1-e^{-\lambda}\right)}, \\
F_{\max }(y \mid \theta, \lambda) & =\frac{e^{-\lambda\left(1+\frac{\theta y}{1+\theta}\right) e^{-\theta y}}-e^{-\lambda}}{1-e^{-\lambda}}, \\
h_{\max }(y \mid \theta, \lambda) & =\frac{\lambda \theta^{2}(1+y) e^{-\left[\theta y+\lambda\left(1+\frac{\theta y}{1+\theta}\right) e^{-\theta y}\right]}}{(1+\theta)\left[1-e^{\left.-\lambda\left(1+\frac{\theta y}{1+\theta}\right) e^{-\theta y}\right]}\right.} .
\end{aligned}
$$

where $\theta>0, \lambda>0$ and $y>0$.

Note that $f_{\max }(0 \mid \theta, \lambda)=\frac{\lambda \theta^{2} e^{-\lambda}}{(\theta+1)\left(1-e^{-\lambda}\right)}$ and $f_{\max }(\infty \mid \theta, \lambda)=0$. For all $\theta>0$ and $\lambda>0$ the probability density function (10), is decreasing or unimodal (see Fig. 4). For values of $\lambda$ close to 1 , the curve resembles the one parameter Lindley distribution, while when $\lambda \longrightarrow \infty$ the curve tends to be symmetric. 
For all $\theta>0$ and $\lambda>0$, the hazard rate function, (12), is increasing (see Fig. 5). Is easy to see that $h_{\max }(0 \mid \theta, \lambda)=f_{\max }(0 \mid \theta, \lambda)=\frac{\lambda \theta^{2} e^{-\lambda}}{(\theta+1)\left(1-e^{-\lambda}\right)}$ and $h_{\max }(\infty \mid \theta, \lambda)=\theta$. Note that $h_{\min }(\infty \mid \theta, \lambda)=h_{\max }(\infty \mid \theta, \lambda)=\theta$.
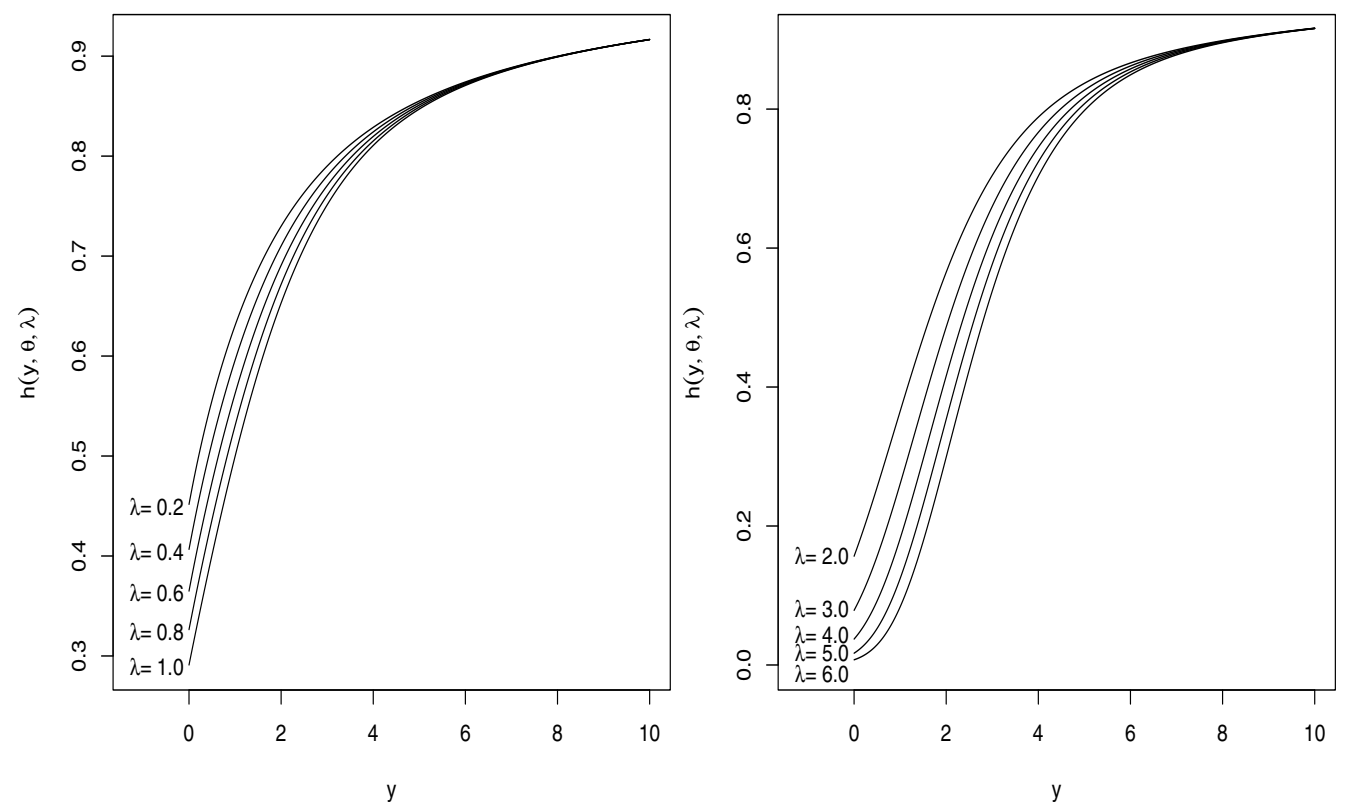

Figure 5 - The zero truncated Lindley-Poisson hazard rate function for different values of the $\lambda$ and $\theta=2.0$ if $Y=\max \left(T_{1}, \ldots, T_{M}\right)$.

Glaser (1980) and Chechile (2003) studied the hazard rate function behavior by the $\eta(y)=$ $\frac{-f^{\prime}(y \mid \theta, \lambda)}{f(y \mid \theta, \lambda)}$ function and its derivative $\eta^{\prime}(y)$. Because of the complexity of such studies, this work only presents the functions $\eta(y)$ and $\eta^{\prime}(y)$. Considering the hazard rate functions (9) and (12), we have:

$$
\begin{aligned}
\eta(y)_{\min } & =\frac{e^{-\theta y}\left[\theta^{2} \lambda(y+1)^{2}+(\theta+1) e^{\theta y}(\theta+\theta y-1)\right]}{(\theta+1)(y+1)}, \\
\eta(y)_{\max } & =-\frac{e^{-\theta y}\left[\theta^{2} \lambda(y+1)^{2}-(\theta+1) e^{\theta y}(\theta+\theta y-1)\right]}{(\theta+1)(y+1)} .
\end{aligned}
$$

and its first derivatives are:

$$
\begin{aligned}
\eta^{\prime}(y)_{\min } & =\frac{e^{-\theta y}\left[(\theta+1) e^{\theta y}-\theta^{2} \lambda(y+1)^{2}(\theta+\theta y-1)\right]}{(\theta+1)(y+1)^{2}}, \\
\eta^{\prime}(y)_{\max } & =-\frac{e^{-\theta y}\left[(\theta+1) e^{\theta y}+\theta^{2} \lambda(y+1)^{2}(\theta+\theta y-1)\right]}{(\theta+1)(y+1)^{2}} .
\end{aligned}
$$


Therefore, the hazard rate function behavior properties of the zero truncated Lindley-Poisson distribution follows from the results in Glaser (1980) and Chechile (2003).

\subsection{Quantile function}

The quantile function of the zero truncated Lindley-Poisson distribution is given by:

$$
F^{-1}(u)=-1-\frac{1}{\theta}-\frac{1}{\theta} W_{-1}\left(-\frac{(\theta+1)}{e^{\theta+1}} \frac{\ln \left(u+e^{\lambda}-u e^{\lambda}\right)}{\lambda}\right)
$$

if $Y=\min \left(T_{1}, \ldots, T_{M}\right)$, where $0<u<1$ and $W_{-1}(\cdot)$ denotes the negative branch of the Lambert $\mathrm{W}$ function (i.e., the solution of the equation $\left.W(z) e^{W(z)}=z\right)$ because $(1+\theta+\theta y)>1$ and $-\frac{(\theta+1)}{e^{\theta+1}} \frac{\ln \left(u+e^{\lambda}-u e^{\lambda}\right)}{\lambda} \in\left(-\frac{1}{e}, 0\right)$. And, the quantile function of the zero truncated LindleyPoisson distribution is given by:

$$
F^{-1}(u)=-1-\frac{1}{\theta}-\frac{1}{\theta} W_{-1}\left(\frac{(\theta+1)}{e^{\theta+1}} \frac{\left[\ln \left(1+u e^{\lambda}-u\right)-\lambda\right]}{\lambda}\right)
$$

if $Y=\max \left(T_{1}, \ldots, T_{M}\right)$, where $0<u<1$ and $W_{-1}(\cdot)$ denotes the negative branch of the Lambert $\mathrm{W}$ function because $(1+\theta+\theta y)>1$ and $\frac{(\theta+1)}{e^{\theta+1}} \frac{\left[\ln \left(1+u e^{\lambda}-u\right)-\lambda\right]}{\lambda} \in\left(-\frac{1}{e}, 0\right)$ (Jodrá, 2010; Ghitany et al., 2012).

Our approach may be generalized in some different ways, for instance, it is important to note that for any probability density function $f_{1}(y \mid \boldsymbol{\theta}), \boldsymbol{\theta}=\left(\theta_{1}, \ldots, \theta_{p}\right)$, and $M \sim$ PoissonTrunc $(\lambda)$ as the discrete distribution, the general marginal probability density function can be written as:

$$
\begin{aligned}
f(y \mid \theta, \lambda) & =\frac{\lambda e^{-\lambda} f_{1}(y \mid \boldsymbol{\theta})}{\left(1-e^{-\lambda}\right)} \sum_{m=1}^{\infty} \frac{\left[\lambda F_{p}(y \mid \boldsymbol{\theta})\right]^{m-1}}{(m-1) !} \\
& =\frac{\lambda f_{1}(y \mid \boldsymbol{\theta}) e^{-\lambda F_{p}(y \mid \boldsymbol{\theta})}}{1-e^{-\lambda}}
\end{aligned}
$$

where $F_{p}(y \mid \boldsymbol{\theta})=F_{1}(y \mid \boldsymbol{\theta})$ when $Y=\min \left(T_{1}, \ldots, T_{M}\right)$ and $F_{p}(y \mid \boldsymbol{\theta})=1-F_{1}(y \mid \boldsymbol{\theta})$ when $Y=\max \left(T_{1}, \ldots, T_{M}\right)$.

From (15), the cumulative distribution, survival and hazard functions for $Y=\min \left(T_{1}, \ldots, T_{M}\right)$ and $Y=\max \left(T_{1}, \ldots, T_{M}\right)$ can be generically written as:

$$
\begin{array}{ll}
F_{\min }(y \mid \boldsymbol{\theta}, \lambda)=\frac{1-e^{-\lambda F_{1}(y \mid \boldsymbol{\theta})}}{1-e^{-\lambda}}, & F_{\max }(y \mid \boldsymbol{\theta}, \lambda)=\frac{e^{-\lambda S_{1}(y \mid \boldsymbol{\theta})}-e^{-\lambda}}{1-e^{-\lambda}}, \\
S_{\min }(y \mid \boldsymbol{\theta}, \lambda)=\frac{e^{-\lambda F_{1}(y \mid \boldsymbol{\theta})}-e^{-\lambda}}{1-e^{-\lambda}}, & S_{\max }(y \mid \boldsymbol{\theta}, \lambda)=\frac{1-e^{-\lambda S_{1}(y \mid \boldsymbol{\theta})}}{1-e^{-\lambda}}, \\
h_{\min }(y \mid \boldsymbol{\theta}, \lambda)=\frac{\lambda f_{1}(y \mid \boldsymbol{\theta}) e^{-\lambda F_{1}(y \mid \boldsymbol{\theta})}}{e^{-\lambda F_{1}(y \mid \boldsymbol{\theta})}-e^{-\lambda}}, & h_{\max }(y \mid \boldsymbol{\theta}, \lambda)=\frac{\lambda f_{1}(y \mid \boldsymbol{\theta}) e^{-\lambda S_{1}(y \mid \boldsymbol{\theta})}}{1-e^{-\lambda F_{1}(y \mid \boldsymbol{\theta})}} .
\end{array}
$$

\section{ESTIMATION METHODS}

In this section, considering the distribution obtained by the composition of distributions we describe six methods used to estimate $\lambda$ and $\theta$. For all methods we consider the case when both $\lambda$ 
and $\theta$ are unknown. This is also considered in the simulation study presented in Section 4. Note that the methods were presented for a general baseline function $f_{1}(y \mid \theta)$.

\subsection{Maximum Likelihood}

Let $\mathbf{y}=\left(y_{1}, \ldots, y_{n}\right)$ be a random sample of $n$ size from the distribution obtained by the composition of distributions with parameters $\lambda$ and $\theta$, the likelihood and $\log$-likelihood function are, respectively:

$$
\begin{gathered}
L(\theta, \lambda \mid \mathbf{y})=\prod_{i=1}^{n} f\left(y_{i} \mid \theta, \lambda\right)=\frac{\lambda^{n} \prod_{i=1}^{n} f_{1}\left(y_{i} \mid \theta\right) e^{-\lambda \sum_{i=1}^{n} F_{p}\left(y_{i} \mid \theta\right)}}{\left(1-e^{-\lambda}\right)^{n}} \\
l(\theta, \lambda \mid \mathbf{y})=n \log \lambda-n \log \left(1-e^{-\lambda}\right)+\sum_{i=1}^{n} \log f_{1}\left(y_{i} \mid \theta\right)-\lambda \sum_{i=1}^{n} F_{p}\left(y_{i} \mid \theta\right),
\end{gathered}
$$

where:

i) $F_{p}\left(y_{i} \mid \theta\right)=F_{1}\left(y_{i} \mid \theta\right)$ if $Y=\min \left(T_{1}, \ldots, T_{M}\right)$

ii) $F_{p}\left(y_{i} \mid \theta\right)=1-F_{1}\left(y_{i} \mid \theta\right)$ if $Y=\max \left(T_{1}, \ldots, T_{M}\right)$.

The maximum likelihood estimates of $\theta$ and $\lambda, \widehat{\theta}_{M L E}$ and $\widehat{\lambda}_{M L E}$ respectively, can be obtained numerically by maximizing the log-likelihood function (17). In this case, the log-likelihood function is maximized by solving numerically $\frac{\partial}{\partial \theta} l(\theta, \lambda \mid \mathbf{y})=0$ and $\frac{\partial}{\partial \lambda} l(\theta, \lambda \mid \mathbf{y})=0$ in $\theta$ and $\lambda$, respectively, where:

$$
\begin{aligned}
\frac{\partial}{\partial \theta} l(\theta, \lambda \mid \mathbf{y}) & =\sum_{i=1}^{n} \frac{f_{1}^{\prime}\left(y_{i} \mid \theta\right)}{f_{1}\left(y_{i} \mid \theta\right)}-\lambda \sum_{i=1}^{n} F_{p}^{\prime}\left(y_{i} \mid \theta\right), \\
\frac{\partial}{\partial \lambda} l(\theta, \lambda \mid \mathbf{y}) & =\frac{n}{\lambda}-\frac{n e^{-\lambda}}{\left(1-e^{-\lambda}\right)}-\sum_{i=1}^{n} F_{p}\left(y_{i} \mid \theta\right),
\end{aligned}
$$

where $f_{1}^{\prime}\left(y_{i} \mid \theta\right)=\frac{\partial}{\partial \theta} f_{1}\left(y_{i} \mid \theta\right)$ and $F_{p}^{\prime}\left(y_{i} \mid \theta\right)=\frac{\partial}{\partial \theta} F_{p}\left(y_{i} \mid \theta\right)$.

\subsection{Ordinary Least-Squares}

Let $y_{1: n}<y_{2: n} \cdots<y_{n: n}$ be the order statistics of a random sample of $n$ size from a distribution with cumulative distribution function $F(y)$. It's well known that:

$$
E\left[F\left(y_{(i: n)}\right)\right]=\frac{i}{n+1} \quad \text { and } \quad \operatorname{Var}\left[F\left(t_{(i: n)}\right)\right]=\frac{i(n-i+1)}{(n+1)^{2}(n+2)} .
$$

For the distribution obtained by the composition process, the least square estimates $\widehat{\theta}_{O L S}$ and $\widehat{\lambda}_{O L S}$ of the parameters $\theta$ and $\lambda$, respectively, are obtained by minimizing the function:

$$
\sum_{i=1}^{n}\left(\frac{1-e^{-\lambda F_{1}\left(y_{i: n} \mid \theta\right)}}{1-e^{-\lambda}}-\frac{i}{n+1}\right)^{2}
$$


when $Y=\min \left(T_{1}, \ldots, T_{M}\right)$, and minimizing

$$
\sum_{i=1}^{n}\left(\frac{e^{-\lambda S_{1}\left(y_{i: n} \mid \theta\right)}-e^{-\lambda}}{1-e^{-\lambda}}-\frac{i}{n+1}\right)^{2},
$$

when $Y=\max \left(T_{1}, \ldots, T_{M}\right)$.

Therefore, if $Y=\min \left(T_{1}, \ldots, T_{M}\right)$, these estimates can also be obtained by solving the nonlinear equations:

$$
\begin{aligned}
& \sum_{i=1}^{n}\left(\frac{1-e^{-\lambda F_{1}\left(y_{i: n} \mid \theta\right)}}{1-e^{-\lambda}}-\frac{i}{n+1}\right) \Delta_{1}\left(y_{i: n} \mid \theta, \lambda\right)=0 \\
& \sum_{i=1}^{n}\left(\frac{1-e^{-\lambda F_{1}\left(y_{i: n} \mid \theta\right)}}{1-e^{-\lambda}}-\frac{i}{n+1}\right) \Delta_{2}\left(y_{i: n} \mid \theta, \lambda\right)=0
\end{aligned}
$$

where:

$$
\begin{aligned}
& \Delta_{1}\left(y_{i: n} \mid \theta, \lambda\right)=\frac{\lambda\left[\frac{\partial}{\partial \theta} F_{1}\left(y_{i: n} \mid \theta\right)\right] e^{-\lambda F_{1}\left(y_{i: n} \mid \theta\right)}}{\left(1-e^{-\lambda}\right)} \\
& \Delta_{2}\left(y_{i: n} \mid \theta, \lambda\right)=\frac{F_{1}\left(y_{i: n} \mid \theta\right) e^{-\lambda F_{1}\left(y_{i: n} \mid \theta\right)}}{\left(1-e^{-\lambda}\right)}-\frac{\left(1-e^{-\lambda F_{1}\left(y_{i: n} \mid \theta\right)}\right) e^{-\lambda}}{\left(1-e^{-\lambda}\right)^{2}}
\end{aligned}
$$

But, if $Y=\max \left(T_{1}, \ldots, T_{M}\right)$, these estimates can also be obtained by solving the nonlinear equations:

$$
\begin{aligned}
& \sum_{i=1}^{n}\left(\frac{e^{-\lambda S_{1}\left(y_{i: n} \mid \theta\right)}-e^{-\lambda}}{1-e^{-\lambda}}-\frac{i}{n+1}\right) \Delta_{1}\left(y_{i: n} \mid \theta, \lambda\right)=0 \\
& \sum_{i=1}^{n}\left(\frac{e^{-\lambda S_{1}\left(y_{i: n} \mid \theta\right)}-e^{-\lambda}}{1-e^{-\lambda}}-\frac{i}{n+1}\right) \Delta_{2}\left(y_{i: n} \mid \theta, \lambda\right)=0
\end{aligned}
$$

where:

$$
\begin{aligned}
& \Delta_{1}\left(y_{i: n} \mid \theta, \lambda\right)=-\frac{\lambda\left[\frac{\partial}{\partial \theta} S_{1}\left(y_{i: n} \mid \theta\right)\right] e^{-\lambda S_{1}\left(y_{i: n} \mid \theta\right)}}{\left(1-e^{-\lambda}\right)} \\
& \Delta_{2}\left(y_{i: n} \mid \theta, \lambda\right)=\frac{-S_{1}\left(y_{i: n} \mid \theta\right) e^{-\lambda S_{1}\left(y_{i: n} \mid \theta\right)}}{\left(1-e^{-\lambda}\right)}-\frac{\left(e^{-\lambda S_{1}\left(y_{i: n} \mid \theta\right)}-e^{-\lambda}\right) e^{-\lambda}}{\left(1-e^{-\lambda}\right)^{2}}
\end{aligned}
$$

Note that $\Delta_{1}$ and $\Delta_{2}$ are derivative from first order distribution function for parameters $\theta$ and $\lambda$, respectively. 


\subsection{Weighted Least-Squares}

The weighted least-squares estimates $\widehat{\theta}_{W L S}$ and $\widehat{\lambda}_{W L S}$ of the parameters $\theta$ and $\lambda$, respectively, are obtained by minimizing the function:

$$
\sum_{i=1}^{n} w_{i}\left(\frac{1-e^{-\lambda F_{1}(y \mid \theta)}}{1-e^{-\lambda}}-\frac{i}{n+1}\right)^{2},
$$

if $Y=\min \left(T_{1}, \ldots, T_{M}\right)$, and minimizing

$$
\sum_{i=1}^{n} w_{i}\left(\frac{e^{-\lambda S_{1}(y \mid \theta)}-e^{-\lambda}}{1-e^{-\lambda}}-\frac{i}{n+1}\right)^{2}
$$

if $Y=\max \left(T_{1}, \ldots, T_{M}\right)$.

The correction factor $w_{i}$ is given by:

$$
w_{i}=\frac{1}{V\left[F\left(y_{(i: n)}\right)\right]}=\frac{(n+1)^{2}(n+2)}{i(n-i+1)} .
$$

Therefore, if $Y=\min \left(T_{1}, \ldots, T_{M}\right)$, these estimates can also be obtained by solving the nonlinear equations:

$$
\begin{aligned}
& \sum_{i=1}^{n} \frac{1}{i(n-i+1)}\left(\frac{1-e^{-\lambda F_{1}(y \mid \theta)}}{1-e^{-\lambda}}-\frac{i}{n+1}\right) \Delta_{1}\left(y_{i: n} \mid \theta, \lambda\right)=0 \\
& \sum_{i=1}^{n} \frac{1}{i(n-i+1)}\left(\frac{1-e^{-\lambda F_{1}(y \mid \theta)}}{1-e^{-\lambda}}-\frac{i}{n+1}\right) \Delta_{2}\left(y_{i: n} \mid \theta, \lambda\right)=0
\end{aligned}
$$

where $\Delta_{1}\left(y_{i: n} \mid \theta, \lambda\right)$ and $\Delta_{2}\left(y_{i: n} \mid \theta, \alpha\right)$ are given by (25) and (26), respectively.

Thus, if $Y=\max \left(T_{1}, \ldots, T_{M}\right)$, these estimates can also be obtained by solving the nonlinear equations:

$$
\begin{aligned}
& \sum_{i=1}^{n} \frac{1}{i(n-i+1)}\left(\frac{e^{-\lambda S_{1}(y \mid \theta)}-e^{-\lambda}}{1-e^{-\lambda}}-\frac{i}{n+1}\right) \Delta_{1}\left(y_{i: n} \mid \theta, \lambda\right)=0 \\
& \sum_{i=1}^{n} \frac{1}{i(n-i+1)}\left(\frac{e^{-\lambda S_{1}(y \mid \theta)}-e^{-\lambda}}{1-e^{-\lambda}}-\frac{i}{n+1}\right) \Delta_{2}\left(y_{i: n} \mid \theta, \lambda\right)=0
\end{aligned}
$$

where $\Delta_{1}\left(y_{i: n} \mid \theta, \lambda\right)$ and $\Delta_{2}\left(y_{i: n} \mid \theta, \alpha\right)$ are given by (29) and (30), respectively.

\subsection{Maximum Product of Spacings}

Cheng \& Amin $(1979,1983)$ introduced the maximum product of spacings (MPS) method as alternative to MLE for the estimation of parameters of continuous univariate distributions. Ranneby (1984) independently developed the same method as an approximation of Kullback-Leibler 
measure of information. In what follows, let $y_{1: n}<y_{2: n}<\cdots<y_{n: n}$ be an ordered random sample drawn from the general model of composition distribution. Are defined as the uniform spacings of the sample the quantities: $D_{1}=F\left(y_{1: n} \mid \theta, \lambda\right), D_{n+1}=1-F\left(t_{n: n} \mid \theta, \lambda\right)$ and $D_{i}=F\left(t_{i: n} \mid \theta, \lambda\right)-F\left(t_{(i-1): n} \mid \theta, \lambda\right), i=2, \ldots, n$. There are $(n+1)$ spacings of the first order.

Following Cheng \& Amin (1983), the maximum product of spacings method consists in finding the values of $\theta$ and $\lambda$ which maximize the geometric mean of the spacings, the MPS statistics, is given by:

$$
G(\theta, \lambda)=\left(\prod_{i=1}^{n+1} D_{i}\right)^{\frac{1}{n+1}}
$$

or, equivalently, its logarithm $H=\log (G)$. Considering $0=F\left(t_{0: n} \mid \theta, \lambda\right)<F\left(y_{1: n} \mid \theta, \lambda\right)<$ $\cdots<F\left(y_{n: n} \mid \theta, \lambda\right)<F\left(y_{(n+1): n} \mid \theta, \lambda\right)=1$ the quantitie $H=\log (G)$ can be calculated as:

$$
H(\theta, \lambda)=\frac{1}{n+1} \sum_{i=1}^{n+1} \log \left[D_{i}\right] .
$$

The estimates for $\theta$ and $\lambda$ can be found solving, respectively in $\theta$ and $\lambda$, the nonlinear equations:

$$
\begin{aligned}
\frac{\partial}{\partial \theta} H(\theta, \lambda) & =\sum_{i=1}^{n+1} \frac{1}{D_{i}} \Delta\left[\frac{\partial}{\partial \theta} F\left(y_{i: n} \mid \theta, \lambda\right)\right]=0 \\
\frac{\partial}{\partial \alpha} H(\theta, \lambda) & =\sum_{i=1}^{n+1} \frac{1}{D_{i}} \Delta\left[\frac{\partial}{\partial \alpha} F\left(y_{i: n} \mid \theta, \lambda\right)\right]=0
\end{aligned}
$$

where $\Delta$ is the first order difference operator.

Cheng \& Amin (1983) showed that maximizing $H$ as a method of parameter estimation is as efficient as MLE estimation and the MPS estimators are consistent under more general conditions than the MLE estimators.

Therefore, if $Y=\min \left(T_{1}, \ldots, T_{M}\right)$, the estimates $\hat{\theta}_{M P S}$ and $\hat{\lambda}_{M P S}$ can be obtained by solving the nonlinear equations:

$$
\begin{aligned}
\frac{\partial}{\partial \theta} H(\theta, \lambda) & =\sum_{i=1}^{n+1} \frac{1}{D_{i}} \Delta\left[\frac{\partial}{\partial \theta}\left(\frac{1-e^{-\lambda F_{1}\left(y_{i: n} \mid \theta\right)}}{1-e^{-\lambda}}\right)\right]=0 \\
\frac{\partial}{\partial \lambda} H(\theta, \lambda) & =\sum_{i=1}^{n+1} \frac{1}{D_{i}} \Delta\left[\frac{\partial}{\partial \lambda}\left(\frac{1-e^{-\lambda F_{1}\left(y_{i: n} \mid \theta\right)}}{1-e^{-\lambda}}\right)\right]=0
\end{aligned}
$$


Thus, if $Y=\max \left(T_{1}, \ldots, T_{M}\right)$, the estimates $\hat{\theta}_{M P S}$ and $\hat{\lambda}_{M P S}$ can be obtained by solving the nonlinear equations:

$$
\begin{aligned}
\frac{\partial}{\partial \theta} H(\theta, \lambda) & =\sum_{i=1}^{n+1} \frac{1}{D_{i}} \Delta\left[\frac{\partial}{\partial \theta}\left(\frac{e^{-\lambda S_{1}\left(y_{i: n} \mid \theta\right)}-e^{-\lambda}}{1-e^{-\lambda}}\right)\right]=0 \\
\frac{\partial}{\partial \lambda} H(\theta, \lambda) & =\sum_{i=1}^{n+1} \frac{1}{D_{i}} \Delta\left[\frac{\partial}{\partial \lambda}\left(\frac{e^{-\lambda S_{1}\left(y_{i: n} \mid \theta\right)}-e^{-\lambda}}{1-e^{-\lambda}}\right)\right]=0
\end{aligned}
$$

\subsection{Minimum distance methods}

In this subsection we present two estimation methods for $\theta$ and $\lambda$ based on the minimization of the goodness-of-fit statistics. This class of statistics is based on the difference between the estimate of the cumulative distribution function and the empirical distribution function (Luceño, 2006).

\subsubsection{Cramér-von-Mises}

The Cramér-von-Mises estimates of the parameters $\widehat{\theta}_{C M}$ and $\widehat{\lambda}_{C M}$, respectively, are obtained by minimizing, in $\theta$ and $\lambda$, the function:

$$
C(\theta, \lambda)=\frac{1}{12 n}+\sum_{i=1}^{n}\left(F\left(y_{i: n} \mid \theta, \lambda\right)-\frac{2 i-1}{2 n}\right)^{2} .
$$

These estimates can also be obtained by solving the nonlinear equations:

$$
\begin{aligned}
& \sum_{i=1}^{n}\left(F\left(y_{i: n} \mid \theta, \lambda\right)-\frac{2 i-1}{2 n}\right) \Delta_{1}\left(y_{i: n} \mid \theta, \lambda\right)=0 \\
& \sum_{i=1}^{n}\left(F\left(y_{i: n} \mid \theta, \lambda\right)-\frac{2 i-1}{2 n}\right) \Delta_{2}\left(y_{i: n} \mid \theta, \lambda\right)=0
\end{aligned}
$$

where $\Delta_{1}(\cdot \mid \theta, \lambda)$ and $\Delta_{2}(\cdot \mid \theta, \lambda)$ are given, respectively, by (25) and (26) if $Y=\min \left(T_{1}\right.$, $\left.\ldots, T_{M}\right)$ and, respectively, by (29) and (30) if $Y=\max \left(T_{1}, \ldots, T_{M}\right)$.

\subsubsection{Anderson-Darling}

The Anderson-Darling estimates of the parameters $\widehat{\theta}_{A D}$ and $\hat{\lambda}_{A D}$, respectively, are obtained by minimizing, with respect to $\theta$ and $\lambda$, the function:

$$
A(\theta, \lambda)=-n-\frac{1}{n} \sum_{i=1}^{n}(2 i-1) \log \left\{F\left(y_{i: n} \mid \theta, \lambda\right)\left[1-F\left(y_{n+1-i: n} \mid \theta, \lambda\right)\right]\right\}
$$


These estimates can also be obtained by solving the nonlinear equations:

$$
\begin{aligned}
& \sum_{i=1}^{n}(2 i-1)\left[\frac{\Delta_{1}\left(y_{i: n} \mid \theta, \lambda\right)}{F\left(y_{i: n} \mid \theta, \lambda\right)}-\frac{\Delta_{1}\left(y_{n+1-i: n} \mid \theta, \lambda\right)}{F\left(y_{n+1-i: n} \mid \theta, \lambda\right)}\right]=0 \\
& \sum_{i=1}^{n}(2 i-1)\left[\frac{\Delta_{2}\left(y_{i: n} \mid \theta, \lambda\right)}{F\left(y_{i: n} \mid \theta, \lambda\right)}-\frac{\Delta_{2}\left(y_{n+1-i: n} \mid \theta, \lambda\right)}{F\left(y_{n+1-i: n} \mid \theta, \lambda\right)}\right]=0
\end{aligned}
$$

where $\Delta_{1}(\cdot \mid \theta, \lambda)$ and $\Delta_{2}(\cdot \mid \theta, \lambda)$ are given, respectively, by (25) and (26) if $Y=\min \left(T_{1}\right.$, $\left.\ldots, T_{M}\right)$ and, respectively, by (29) and (30) if $Y=\max \left(T_{1}, \ldots, T_{M}\right)$.

\section{SIMULATION STUDY}

In this section we present results of some numerical experiments to compare the performance of the different estimation methods discussed in the previous section. We have taken sample sizes $n=20,50,100$ and 200, $\theta=1.0$ and $\lambda=0.5,1.0,2.0,3.0$ and 5.0. For each combination $(n, \theta, \lambda)$ we have generated $B=500,000$ pseudo random samples from the zero truncated Lindley-Poisson distribution.

The estimates were obtained in $O x$ version 6.20 (Doornik, 2007) using MaxBFGS function in MLE, OLS, WLS, MPS, CM and AD methods. For each estimate we computed the bias, the root mean-squared error, the average absolute difference between the true and estimate distributions functions and the maximum absolute difference between the true and estimate distributions functions, respectively, as:

$$
\begin{aligned}
\operatorname{Bias}(\hat{\theta}) & =\frac{1}{B} \sum_{i=1}^{B}\left(\hat{\theta}_{i}-\theta\right), \quad \operatorname{Bias}(\hat{\lambda})=\frac{1}{B} \sum_{i=1}^{B}\left(\hat{\lambda}_{i}-\lambda\right), \\
\operatorname{RMSE}(\hat{\theta}) & =\sqrt{\frac{1}{B} \sum_{i=1}^{B}\left(\hat{\theta}_{i}-\theta\right)^{2}}, \quad \operatorname{RMSE}(\hat{\lambda})=\sqrt{\frac{1}{B} \sum_{i=1}^{B}\left(\hat{\lambda}_{i}-\lambda\right)^{2},} \\
D_{a b s} & =\frac{1}{B \times n} \sum_{i=1}^{B} \sum_{j=1}^{n}\left|F\left(y_{i j} \mid \theta, \lambda\right)-F\left(y_{i j} \mid \hat{\theta}, \hat{\lambda}\right)\right|, \\
D_{\max } & =\frac{1}{B} \sum_{i=1}^{B} \max _{j}\left|F\left(y_{i j} \mid \theta, \lambda\right)-F\left(y_{i j} \mid \hat{\theta}, \hat{\lambda}\right)\right| .
\end{aligned}
$$

In Tables 1, 2, 3, 4 and 5 we show the calculated values of (52)-(55). The superscript values indicate the rank obtained by each of the methods considered, and the total line shows the global rank for each method based on measures (52)-(55).

For the simulations, the MLE method proved to be the most efficient for estimate the parameters of zero truncated Lindley-Poisson distribution to $Y=\min \left(T_{1}, \ldots, T_{M}\right)$ when $\lambda=0.5$ and $\lambda=1.0$. For $\lambda=2.0,3.0$ and 5.0, the OLS, in general, proved to be better. 
Table 1 - Simulations results for $\theta=1.0$ and $\lambda=0.5$.

\begin{tabular}{|c|c|c|c|c|c|c|c|}
\hline $\mathrm{n}$ & Qtd & MLE & MPS & OLS & WLS & $\mathrm{CM}$ & $\mathrm{AD}$ \\
\hline \multirow{6}{*}{20} & $\overline{\operatorname{Bias}(\theta)}$ & $-0.1270^{1}$ & $-0.2704^{6}$ & $-0.2096^{4}$ & $-0.2230^{5}$ & $-0.1692^{2}$ & $-0.1978^{3}$ \\
\hline & $R M S E(\theta)$ & $0.2791^{2}$ & $0.3549^{6}$ & $0.2842^{3}$ & $0.3019^{5}$ & $0.2688^{1}$ & $0.2975^{4}$ \\
\hline & $\operatorname{Bias}(\lambda)$ & $1.0086^{1}$ & $1.6417^{6}$ & $1.2324^{3}$ & $1.3480^{5}$ & $1.1070^{2}$ & $1.3152^{4}$ \\
\hline & $R M S E(\lambda)$ & $1.6054^{3}$ & $2.0924^{6}$ & $1.4874^{2}$ & $1.6620^{4}$ & $1.4262^{1}$ & $1.7573^{5}$ \\
\hline & $D_{a b s}$ & $0.0472^{2}$ & $0.0469^{1}$ & $0.0501^{6}$ & $0.0489^{4}$ & $0.0497^{5}$ & $0.0483^{3}$ \\
\hline & $D_{\max }$ & $0.0739^{2}$ & $0.0710^{1}$ & $0.0776^{5}$ & $0.0760^{4}$ & $0.0786^{6}$ & $0.0757^{3}$ \\
\hline & Total & $11^{1}$ & $26^{5}$ & $23^{4}$ & $27^{6}$ & $17^{2}$ & $22^{3}$ \\
\hline $\mathrm{n}$ & Qtd & MLE & MPS & OLS & WLS & $\mathrm{CM}$ & $\mathrm{AD}$ \\
\hline \multirow{7}{*}{50} & $\operatorname{Bias}(\theta)$ & $-0.1199^{1}$ & $-0.2432^{6}$ & $-0.1771^{5}$ & $-0.1771^{4}$ & $-0.1497^{2}$ & $-0.1567^{3}$ \\
\hline & $R M S E(\theta)$ & $0.2453^{2}$ & $0.3336^{6}$ & $0.2475^{3}$ & $0.2580^{5}$ & $0.2337^{1}$ & $0.2487^{4}$ \\
\hline & $\operatorname{Bias}(\lambda)$ & $0.8475^{1}$ & $1.5288^{6}$ & $0.9810^{4}$ & $1.0301^{5}$ & $0.8742^{2}$ & $0.9570^{3}$ \\
\hline & $R M S E(\lambda)$ & $1.5436^{5}$ & $2.1627^{6}$ & $1.3102^{2}$ & $1.4556^{4}$ & $1.2433^{1}$ & $1.4354^{3}$ \\
\hline & $D_{a b s}$ & $0.0309^{2}$ & $0.0306^{1}$ & $0.0323^{6}$ & $0.0316^{4}$ & $0.0322^{5}$ & $0.0315^{3}$ \\
\hline & $D_{\max }$ & $0.0483^{2}$ & $0.0474^{1}$ & $0.0508^{5}$ & $0.0498^{4}$ & $0.0510^{6}$ & $0.0496^{3}$ \\
\hline & Total & $13^{1}$ & $26^{5}$ & $25^{4}$ & $26^{5}$ & $17^{2}$ & $19^{3}$ \\
\hline \multirow[t]{2}{*}{$\mathrm{n}$} & Qtd & MLE & MPS & OLS & WLS & $\mathrm{CM}$ & $\mathrm{AD}$ \\
\hline & $\operatorname{Bias}(\theta)$ & $-0.0897^{1}$ & $-0.1926^{6}$ & $-0.1412^{5}$ & $-0.1263^{4}$ & $-0.1208^{3}$ & $-0.1149^{2}$ \\
\hline \multirow{6}{*}{100} & $R M S E(\theta)$ & $0.2063^{3}$ & $0.2941^{6}$ & $0.2163^{5}$ & $0.2129^{4}$ & $0.2046^{1}$ & $0.2053^{2}$ \\
\hline & $\operatorname{Bias}(\lambda)$ & $0.6062^{1}$ & $1.2199^{6}$ & $0.7576^{5}$ & $0.7138^{4}$ & $0.6722^{3}$ & $0.6668^{2}$ \\
\hline & $R M S E(\lambda)$ & $1.3038^{5}$ & $1.9712^{6}$ & $1.1311^{2}$ & $1.1714^{4}$ & $1.0695^{1}$ & $1.1418^{3}$ \\
\hline & $D_{a b s}$ & $0.0223^{2}$ & $0.0223^{1}$ & $0.0233^{6}$ & $0.0227^{4}$ & $0.0232^{5}$ & $0.0227^{3}$ \\
\hline & $D_{\max }$ & $0.0351^{1}$ & $0.0352^{2}$ & $0.0372^{6}$ & $0.0362^{4}$ & $0.0371^{5}$ & $0.0362^{3}$ \\
\hline & Total & $13^{1}$ & $27^{5}$ & $29^{6}$ & $24^{4}$ & $18^{3}$ & $15^{2}$ \\
\hline $\mathrm{n}$ & Qtd & MLE & MPS & OLS & WLS & $\mathrm{CM}$ & $\mathrm{AD}$ \\
\hline \multirow{7}{*}{200} & $\operatorname{Bias}(\theta)$ & $-0.0532^{1}$ & $-0.1255^{6}$ & $-0.0989^{5}$ & $-0.0770^{3}$ & $-0.0849^{4}$ & $-0.0718^{2}$ \\
\hline & $R M S E(\theta)$ & $0.1569^{1}$ & $0.2290^{6}$ & $0.1786^{5}$ & $0.1617^{3}$ & $0.1701^{4}$ & $0.1580^{2}$ \\
\hline & $\operatorname{Bias}(\lambda)$ & $0.3469^{1}$ & $0.7737^{6}$ & $0.5174^{5}$ & $0.4219^{3}$ & $0.4576^{4}$ & $0.4002^{2}$ \\
\hline & $R M S E(\lambda)$ & $0.9570^{5}$ & $1.5332^{6}$ & $0.9079^{4}$ & $0.8508^{2}$ & $0.8606^{3}$ & $0.8323^{1}$ \\
\hline & $D_{a b s}$ & $0.0161^{1}$ & $0.0162^{2}$ & $0.0168^{6}$ & $0.0164^{3}$ & $0.0167^{5}$ & $0.0164^{4}$ \\
\hline & $D_{\max }$ & $0.0257^{1}$ & $0.0261^{2}$ & $0.0273^{6}$ & $0.0265^{4}$ & $0.0272^{5}$ & $0.0264^{3}$ \\
\hline & Total & $10^{1}$ & $28^{5}$ & $31^{6}$ & $18^{3}$ & $25^{4}$ & $14^{2}$ \\
\hline
\end{tabular}

Qtd: measures (52)-(55)

In Tables 6, 7, 8, 9 and 10 we show the calculated values of (52)-(55). The superscript values indicate the rank obtained by each of the methods considered, and the total line shows the global rank for each method based on measures (52)-(55).

In general, the MPS method proved to be the best method to estimate the parameters of the zero truncated Lindley-Poisson distribution to $Y=\max \left(T_{1}, \ldots, T_{M}\right)$. The MLE method showed the worst results even for the large sample size. For future work, further study the zero truncated 
Table 2 - Simulations results for $\theta=1.0$ and $\lambda=1.0$.

\begin{tabular}{|c|c|c|c|c|c|c|c|}
\hline $\mathrm{n}$ & Qtd & MLE & MPS & OLS & WLS & $\mathrm{CM}$ & $\mathrm{AD}$ \\
\hline \multirow{6}{*}{20} & $\operatorname{Bias}(\theta)$ & $-0.0539^{1}$ & $-0.2078^{6}$ & $-0.1314^{4}$ & $-0.1538^{5}$ & $-0.0880^{2}$ & $-0.1300^{3}$ \\
\hline & $R M S E(\theta)$ & $0.2864^{5}$ & $0.3274^{6}$ & $0.2549^{1}$ & $0.2787^{3}$ & $0.2551^{2}$ & $0.2837^{4}$ \\
\hline & $\operatorname{Bias}(\lambda)$ & $0.6371^{1}$ & $1.2563^{6}$ & $0.8397^{3}$ & $0.9961^{5}$ & $0.7165^{2}$ & $0.9710^{4}$ \\
\hline & $R M S E(\lambda)$ & $1.4101^{3}$ & $1.7781^{6}$ & $1.1841^{2}$ & $1.4146^{4}$ & $1.1625^{1}$ & $1.5388^{5}$ \\
\hline & $D_{a b s}$ & $0.0471^{1}$ & $0.0474^{2}$ & $0.0503^{6}$ & $0.0493^{4}$ & $0.0498^{5}$ & $0.0483^{3}$ \\
\hline & $D_{\max }$ & $0.0732^{2}$ & $0.0712^{1}$ & $0.0769^{5}$ & $0.0755^{4}$ & $0.0778^{6}$ & $0.0749^{3}$ \\
\hline & Total & $13^{1}$ & $27^{6}$ & $21^{3}$ & $25^{5}$ & $18^{2}$ & $22^{4}$ \\
\hline $\mathrm{n}$ & Qtd & MLE & MPS & OLS & WLS & $\mathrm{CM}$ & $\mathrm{AD}$ \\
\hline \multirow{7}{*}{50} & $\operatorname{Bias}(\theta)$ & $-0.0596^{1}$ & $-0.1953^{6}$ & $-0.1049^{4}$ & $-0.1218^{5}$ & $-0.0748^{2}$ & $-0.0980^{3}$ \\
\hline & $R M S E(\theta)$ & $0.2496^{5}$ & $0.3128^{6}$ & $0.2208^{2}$ & $0.2426^{4}$ & $0.2180^{1}$ & $0.2408^{3}$ \\
\hline & $\operatorname{Bias}(\lambda)$ & $0.5523^{2}$ & $1.2346^{6}$ & $0.6322^{3}$ & $0.7659^{5}$ & $0.5252^{1}$ & $0.6770^{4}$ \\
\hline & $R M S E(\lambda)$ & $1.4219^{5}$ & $1.9128^{6}$ & $1.0698^{2}$ & $1.3248^{4}$ & $1.0372^{1}$ & $1.2955^{3}$ \\
\hline & $D_{a b s}$ & $0.0307^{2}$ & $0.0307^{1}$ & $0.0322^{6}$ & $0.0316^{4}$ & $0.0322^{5}$ & $0.0314^{3}$ \\
\hline & $D_{\max }$ & $0.0478^{2}$ & $0.0470^{1}$ & $0.0497^{5}$ & $0.0490^{4}$ & $0.0501^{6}$ & $0.0487^{3}$ \\
\hline & Total & $17^{2}$ & $26^{5}$ & $22^{4}$ & $26^{5}$ & $16^{1}$ & $19^{3}$ \\
\hline $\mathrm{n}$ & Qtd & MLE & MPS & OLS & WLS & $\mathrm{CM}$ & $\mathrm{AD}$ \\
\hline \multirow{7}{*}{100} & $\operatorname{Bias}(\theta)$ & $-0.0439^{1}$ & $-0.1628^{6}$ & $-0.0781^{4}$ & $-0.0795^{5}$ & $-0.0555^{2}$ & $-0.0670^{3}$ \\
\hline & $R M S E(\theta)$ & $0.2223^{5}$ & $0.2896^{6}$ & $0.2031^{2}$ & $0.2150^{4}$ & $0.1998^{1}$ & $0.2119^{3}$ \\
\hline & $\operatorname{Bias}(\lambda)$ & $0.4063^{2}$ & $1.0543^{6}$ & $0.4728^{4}$ & $0.5086^{5}$ & $0.3852^{1}$ & $0.4617^{3}$ \\
\hline & $R M S E(\lambda)$ & $1.2928^{5}$ & $1.8326^{6}$ & $0.9841^{2}$ & $1.1213^{4}$ & $0.9536^{1}$ & $1.1111^{3}$ \\
\hline & $D_{a b s}$ & $0.0223^{2}$ & $0.0222^{1}$ & $0.0231^{6}$ & $0.0227^{4}$ & $0.0231^{5}$ & $0.0226^{3}$ \\
\hline & $D_{\max }$ & $0.0351^{2}$ & $0.0348^{1}$ & $0.0362^{5}$ & $0.0356^{4}$ & $0.0364^{6}$ & $0.0356^{3}$ \\
\hline & Total & $17^{2}$ & $26^{5}$ & $23^{4}$ & $26^{5}$ & $16^{1}$ & $18^{3}$ \\
\hline $\mathrm{n}$ & Qtd & MLE & MPS & OLS & WLS & $\mathrm{CM}$ & $\mathrm{AD}$ \\
\hline \multirow{7}{*}{200} & $\overline{\operatorname{Bias}(\theta)}$ & $-0.0245^{1}$ & $-0.1194^{6}$ & $-0.0508^{5}$ & $-0.0444^{4}$ & $-0.0340^{2}$ & $-0.0383^{3}$ \\
\hline & $R M S E(\theta)$ & $0.1894^{5}$ & $0.2513^{6}$ & $0.1848^{4}$ & $0.1847^{3}$ & $0.1823^{1}$ & $0.1824^{2}$ \\
\hline & $\operatorname{Bias}(\lambda)$ & $0.2488^{1}$ & $0.7783^{6}$ & $0.3208^{5}$ & $0.3012^{4}$ & $0.2529^{2}$ & $0.2770^{3}$ \\
\hline & $R M S E(\lambda)$ & $1.0876^{5}$ & $1.5969^{6}$ & $0.8812^{2}$ & $0.9308^{4}$ & $0.8560^{1}$ & $0.9233^{3}$ \\
\hline & $D_{a b s}$ & $0.0162^{2}$ & $0.0162^{1}$ & $0.0167^{5}$ & $0.0164^{4}$ & $0.0167^{6}$ & $0.0164^{3}$ \\
\hline & $D_{\max }$ & $0.0259^{2}$ & $0.0258^{1}$ & $0.0267^{5}$ & $0.0262^{4}$ & $0.0268^{6}$ & $0.0262^{3}$ \\
\hline & Total & $16^{1}$ & $26^{5}$ & $26^{5}$ & $23^{4}$ & $18^{3}$ & $17^{2}$ \\
\hline
\end{tabular}

Qtd: measures (52)-(55)

Lindley-Poisson distribution to $Y=\max \left(T_{1}, \ldots, T_{M}\right)$ to understand why the MLE method was not as good would be very relevant.

For $\lambda=0.5$ and 1.0 the MPS method had the highest rank and AD method the second. For $\lambda=3.0$ and 5.0 the AD method was the best, the MPS rank was the second one, only when $n=20$ the MPS was better, and for $\lambda=2.0$, the MPS was better for $n=20$ and 50 while the AD was better for $n=100$ and 200 . 
Table 3 - Simulations results for $\theta=1.0$ and $\lambda=2.0$.

\begin{tabular}{|c|c|c|c|c|c|c|c|}
\hline $\mathrm{n}$ & Qtd & MLE & MPS & OLS & WLS & $\mathrm{CM}$ & $\mathrm{AD}$ \\
\hline \multirow{6}{*}{20} & $\operatorname{Bias}(\theta)$ & $0.1166^{6}$ & $-0.0761^{5}$ & $0.0150^{2}$ & $-0.0271^{3}$ & $0.0546^{4}$ & $-0.0085^{1}$ \\
\hline & $R M S E(\theta)$ & $0.3954^{6}$ & $0.3298^{4}$ & $0.2784^{1}$ & $0.3196^{3}$ & $0.3157^{2}$ & $0.3352^{5}$ \\
\hline & $\operatorname{Bias}(\lambda)$ & $-0.0191^{1}$ & $0.6555^{6}$ & $0.2555^{3}$ & $0.5789^{4}$ & $0.1911^{2}$ & $0.5801^{5}$ \\
\hline & $R M S E(\lambda)$ & $1.4987^{3}$ & $1.6339^{4}$ & $1.0738^{1}$ & $1.6389^{5}$ & $1.2154^{2}$ & $1.7052^{6}$ \\
\hline & $D_{a b s}$ & $0.0477^{1}$ & $0.0487^{3}$ & $0.0505^{6}$ & $0.0505^{5}$ & $0.0499^{4}$ & $0.0486^{2}$ \\
\hline & $D_{\max }$ & $0.0746^{2}$ & $0.0742^{1}$ & $0.0776^{4}$ & $0.0777^{5}$ & $0.0782^{6}$ & $0.0759^{3}$ \\
\hline & Total & $19^{2}$ & $23^{5}$ & $17^{1}$ & $25^{6}$ & $20^{3}$ & $22^{4}$ \\
\hline $\mathrm{n}$ & Qtd & MLE & MPS & OLS & WLS & $\mathrm{CM}$ & $\mathrm{AD}$ \\
\hline \multirow{7}{*}{50} & $\operatorname{Bias}(\theta)$ & $0.0734^{5}$ & $-0.0902^{6}$ & $0.0068^{2}$ & $-0.0474^{4}$ & $0.0346^{3}$ & $0.0042^{1}$ \\
\hline & $R M S E(\theta)$ & $0.3316^{6}$ & $0.3046^{5}$ & $0.2465^{1}$ & $0.2781^{3}$ & $0.2722^{2}$ & $0.2920^{4}$ \\
\hline & $\operatorname{Bias}(\lambda)$ & $0.0558^{1}$ & $0.7365^{6}$ & $0.2347^{3}$ & $0.6228^{5}$ & $0.1677^{2}$ & $0.3702^{4}$ \\
\hline & $R M S E(\lambda)$ & $1.5182^{3}$ & $1.6644^{5}$ & $1.1754^{1}$ & $1.7368^{6}$ & $1.2564^{2}$ & $1.5471^{4}$ \\
\hline & $D_{a b s}$ & $0.0313^{1}$ & $0.0315^{2}$ & $0.0325^{6}$ & $0.0320^{4}$ & $0.0324^{5}$ & $0.0316^{3}$ \\
\hline & $D_{\max }$ & $0.0493^{2}$ & $0.0490^{1}$ & $0.0501^{4}$ & $0.0502^{5}$ & $0.0506^{6}$ & $0.0496^{3}$ \\
\hline & Total & $18^{2}$ & $25^{5}$ & $17^{1}$ & $27^{6}$ & $20^{4}$ & $19^{3}$ \\
\hline \multirow[t]{4}{*}{$\mathrm{n}$} & Qtd & MLE & MPS & OLS & WLS & $\mathrm{CM}$ & $\mathrm{AD}$ \\
\hline & $\operatorname{Bias}(\theta)$ & $0.0572^{5}$ & $-0.0879^{6}$ & $0.0010^{1}$ & $-0.0335^{4}$ & $0.0206^{2}$ & $0.0237^{3}$ \\
\hline & $R M S E(\theta)$ & $0.2973^{6}$ & $0.2882^{5}$ & $0.2444^{1}$ & $0.2781^{4}$ & $0.2633^{2}$ & $0.2745^{3}$ \\
\hline & $\operatorname{Bias}(\lambda)$ & $0.0525^{1}$ & $0.7127^{6}$ & $0.2638^{4}$ & $0.5225^{5}$ & $0.2153^{3}$ & $0.1933^{2}$ \\
\hline \multirow[t]{4}{*}{100} & $R M S E(\lambda)$ & $1.4109^{4}$ & $1.6166^{5}$ & $1.2692^{1}$ & $1.6619^{6}$ & $1.3338^{2}$ & $1.3535^{3}$ \\
\hline & $D_{a b s}$ & $0.0227^{1}$ & $0.0227^{2}$ & $0.0232^{5}$ & $0.0232^{4}$ & $0.0233^{6}$ & $0.0230^{3}$ \\
\hline & $D_{\max }$ & $0.0361^{2}$ & $0.0360^{1}$ & $0.0363^{3}$ & $0.0368^{6}$ & $0.0367^{5}$ & $0.0365^{4}$ \\
\hline & Total & $19^{3}$ & $25^{5}$ & $15^{1}$ & $29^{6}$ & $20^{4}$ & $18^{2}$ \\
\hline \multirow[t]{4}{*}{$\mathrm{n}$} & Qtd & MLE & MPS & OLS & WLS & $\mathrm{CM}$ & $\mathrm{AD}$ \\
\hline & $\operatorname{Bias}(\theta)$ & $0.0393^{5}$ & $-0.0813^{6}$ & $-0.0012^{1}$ & $-0.0084^{2}$ & $0.0125^{3}$ & $0.0314^{4}$ \\
\hline & $R M S E(\theta)$ & $0.2584^{4}$ & $0.2624^{5}$ & $0.2393^{1}$ & $0.2627^{6}$ & $0.2538^{3}$ & $0.2463^{2}$ \\
\hline & $\operatorname{Bias}(\lambda)$ & $0.0586^{1}$ & $0.6369^{6}$ & $0.2570^{4}$ & $0.3263^{5}$ & $0.2243^{3}$ & $0.0798^{2}$ \\
\hline \multirow[t]{4}{*}{200} & $\operatorname{RMSE}(\lambda)$ & $1.2536^{3}$ & $1.4862^{6}$ & $1.2508^{2}$ & $1.4170^{5}$ & $1.3038^{4}$ & $1.1280^{1}$ \\
\hline & $D_{a b s}$ & $0.0164^{2}$ & $0.0164^{1}$ & $0.0167^{5}$ & $0.0167^{3}$ & $0.0168^{6}$ & $0.0167^{4}$ \\
\hline & $D_{\max }$ & $0.0262^{1}$ & $0.0263^{2}$ & $0.0266^{3}$ & $0.0269^{5}$ & $0.0270^{6}$ & $0.0267^{4}$ \\
\hline & Total & $16^{1}$ & $26^{5}$ & $16^{1}$ & $26^{5}$ & $25^{4}$ & $17^{3}$ \\
\hline
\end{tabular}

Qtd: measures (52)-(55)

\section{REAL DATA APPLICATION}

In this section we fit the zero truncated Lindley-Poisson distribution (LP) to a real data set. For comparison, we also have considered four alternative models: the one parameter Lindley distribution (L) $f(y \mid \theta)=\frac{\theta^{2}}{1+\theta}(1+y) e^{-\theta y}$, the weighted Lindley distribution (WL)

$$
f(y \mid \theta, \lambda)=\frac{\theta^{\lambda+1}}{(\theta+\lambda) \Gamma(\lambda)} y^{\lambda-1}(1+y) e^{-\theta y},
$$


Table 4 - Simulations results for $\theta=1.0$ and $\lambda=3.0$.

\begin{tabular}{|c|c|c|c|c|c|c|c|}
\hline $\mathrm{n}$ & Qtd & MLE & MPS & OLS & WLS & $\mathrm{CM}$ & $\mathrm{AD}$ \\
\hline \multirow{6}{*}{20} & $\operatorname{Bias}(\theta)$ & $0.1037^{5}$ & $-0.0823^{4}$ & $0.0490^{3}$ & $-0.0358^{2}$ & $0.0226^{1}$ & $-0.1151^{6}$ \\
\hline & $R M S E(\theta)$ & $0.5168^{6}$ & $0.3539^{5}$ & $0.3152^{1}$ & $0.3266^{2}$ & $0.3389^{3}$ & $0.3473^{4}$ \\
\hline & $\operatorname{Bias}(\lambda)$ & $0.7798^{4}$ & $0.9108^{5}$ & $0.0456^{1}$ & $0.6362^{3}$ & $0.3493^{2}$ & $1.2883^{6}$ \\
\hline & $R M S E(\lambda)$ & $4.1180^{6}$ & $2.9045^{5}$ & $1.5550^{1}$ & $2.2861^{3}$ & $1.8862^{2}$ & $2.7833^{4}$ \\
\hline & $D_{a b s}$ & $0.0490^{3}$ & $0.0522^{6}$ & $0.0496^{5}$ & $0.0495^{4}$ & $0.0483^{1}$ & $0.0489^{2}$ \\
\hline & $D_{\max }$ & $0.0754^{5}$ & $0.0777^{6}$ & $0.0745^{2}$ & $0.0750^{4}$ & $0.0744^{1}$ & $0.0749^{3}$ \\
\hline & Total & $29^{5}$ & $31^{6}$ & $13^{2}$ & $18^{3}$ & $10^{1}$ & $25^{4}$ \\
\hline $\mathrm{n}$ & Qtd & MLE & MPS & OLS & WLS & $\mathrm{CM}$ & $\mathrm{AD}$ \\
\hline \multirow{7}{*}{50} & $\operatorname{Bias}(\theta)$ & $-0.0200^{1}$ & $-0.1466^{4}$ & $-0.0477^{2}$ & $-0.1856^{6}$ & $-0.0719^{3}$ & $-0.1845^{5}$ \\
\hline & $R M S E(\theta)$ & $0.4429^{6}$ & $0.3381^{5}$ & $0.2893^{1}$ & $0.3337^{3}$ & $0.3076^{2}$ & $0.3346^{4}$ \\
\hline & $\operatorname{Bias}(\lambda)$ & $1.3368^{3}$ & $1.3400^{4}$ & $0.5880^{1}$ & $1.6653^{5}$ & $0.8170^{2}$ & $1.6955^{6}$ \\
\hline & $R M S E(\lambda)$ & $4.1031^{6}$ & $3.0049^{5}$ & $1.9332^{1}$ & $2.9905^{4}$ & $2.1363^{2}$ & $2.9661^{3}$ \\
\hline & $D_{a b s}$ & $0.0342^{4}$ & $0.0356^{6}$ & $0.0333^{2}$ & $0.0345^{5}$ & $0.0330^{1}$ & $0.0334^{3}$ \\
\hline & $D_{\max }$ & $0.0535^{4}$ & $0.0543^{6}$ & $0.0509^{1}$ & $0.0538^{5}$ & $0.0513^{2}$ & $0.0523^{3}$ \\
\hline & Total & $24^{3}$ & $30^{6}$ & $8^{1}$ & $28^{5}$ & $12^{2}$ & $24^{3}$ \\
\hline \multirow[t]{2}{*}{$\mathrm{n}$} & Qtd & MLE & MPS & OLS & WLS & $\mathrm{CM}$ & $\mathrm{AD}$ \\
\hline & $\operatorname{Bias}(\theta)$ & $-0.0825^{1}$ & $-0.1767^{4}$ & $-0.1367^{2}$ & $-0.2656^{6}$ & $-0.1612^{3}$ & $-0.2358^{5}$ \\
\hline \multirow{6}{*}{100} & $R M S E(\theta)$ & $0.4027^{6}$ & $0.3275^{3}$ & $0.3044^{1}$ & $0.3553^{5}$ & $0.3195^{2}$ & $0.3409^{4}$ \\
\hline & $\operatorname{Bias}(\lambda)$ & $1.4136^{2}$ & $1.4163^{3}$ & $1.1963^{1}$ & $2.2229^{6}$ & $1.4221^{4}$ & $1.9891^{5}$ \\
\hline & $R M S E(\lambda)$ & $3.5852^{6}$ & $2.6768^{3}$ & $2.3528^{1}$ & $3.2513^{5}$ & $2.5363^{2}$ & $3.0507^{4}$ \\
\hline & $D_{a b s}$ & $0.0272^{4}$ & $0.0278^{5}$ & $0.0256^{2}$ & $0.0280^{6}$ & $0.0255^{1}$ & $0.0266^{3}$ \\
\hline & $D_{\max }$ & $0.0431^{4}$ & $0.0432^{5}$ & $0.0402^{1}$ & $0.0448^{6}$ & $0.0406^{2}$ & $0.0426^{3}$ \\
\hline & Total & $23^{3}$ & $23^{3}$ & $8^{1}$ & $34^{6}$ & $14^{2}$ & $24^{5}$ \\
\hline $\mathrm{n}$ & Qtd & MLE & MPS & OLS & WLS & $\mathrm{CM}$ & $\mathrm{AD}$ \\
\hline \multirow{7}{*}{200} & $\operatorname{Bias}(\theta)$ & $-0.1245^{1}$ & $-0.1966^{2}$ & $-0.2086^{3}$ & $-0.3272^{6}$ & $-0.2270^{4}$ & $-0.2816^{5}$ \\
\hline & $R M S E(\theta)$ & $0.3542^{5}$ & $0.3097^{1}$ & $0.3175^{2}$ & $0.3745^{6}$ & $0.3290^{3}$ & $0.3482^{4}$ \\
\hline & $\operatorname{Bias}(\lambda)$ & $1.2625^{1}$ & $1.3840^{2}$ & $1.6517^{3}$ & $2.6237^{6}$ & $1.8255^{4}$ & $2.2034^{5}$ \\
\hline & $R M S E(\lambda)$ & $2.7205^{4}$ & $2.2294^{1}$ & $2.5545^{2}$ & $3.3455^{6}$ & $2.6941^{3}$ & $3.0007^{5}$ \\
\hline & $D_{a b s}$ & $0.0222^{3}$ & $0.0226^{4}$ & $0.0209^{1}$ & $0.0245^{6}$ & $0.0209^{2}$ & $0.0233^{5}$ \\
\hline & $D_{\max }$ & $0.0353^{3}$ & $0.0355^{4}$ & $0.0336^{1}$ & $0.0398^{6}$ & $0.0340^{2}$ & $0.0377^{5}$ \\
\hline & Total & $17^{3}$ & $14^{2}$ & $12^{1}$ & $36^{6}$ & $18^{4}$ & $29^{5}$ \\
\hline
\end{tabular}

Qtd: measures (52)-(55)

the exponentiated or generalized Lindley distribution (EL)

$$
f(y \mid \theta, \lambda)=\frac{\lambda \theta^{2}}{1+\theta}(1+y) e^{-\theta y}\left[1-\left(1+\frac{\theta y}{1+\theta}\right) e^{-\theta y}\right]^{\lambda-1}
$$

and the power Lindley distribution (PL)

$$
f(y \mid \theta, \lambda)=\frac{\lambda \theta^{2}}{1+\theta}\left(1+y^{\lambda}\right) y^{\lambda-1} e^{-\theta y^{\lambda}} .
$$


Table 5 - Simulations results for $\theta=1.0$ and $\lambda=5.0$.

\begin{tabular}{|c|c|c|c|c|c|c|c|}
\hline $\mathrm{n}$ & Qtd & MLE & MPS & OLS & WLS & $\mathrm{CM}$ & $\mathrm{AD}$ \\
\hline \multirow{6}{*}{20} & $\overline{\operatorname{Bias}(\theta)}$ & $-0.1802^{6}$ & $-0.1065^{4}$ & $0.0306^{2}$ & $-0.0661^{3}$ & $-0.0110^{1}$ & $-0.1363^{5}$ \\
\hline & $R M S E(\theta)$ & $0.4768^{6}$ & $0.3699^{1}$ & $0.3837^{2}$ & $0.3857^{3}$ & $0.4002^{4}$ & $0.4139^{5}$ \\
\hline & $\operatorname{Bias}(\lambda)$ & $3.0350^{6}$ & $0.7518^{4}$ & $-0.4789^{3}$ & $0.3519^{2}$ & $-0.0335^{1}$ & $1.1322^{5}$ \\
\hline & $R M S E(\lambda)$ & $6.6507^{6}$ & $3.8766^{5}$ & $2.4045^{1}$ & $2.9953^{3}$ & $2.5465^{2}$ & $3.3829^{4}$ \\
\hline & $D_{a b s}$ & $0.0745^{5}$ & $0.0790^{6}$ & $0.0702^{2}$ & $0.0734^{4}$ & $0.0668^{1}$ & $0.0731^{3}$ \\
\hline & $D_{\max }$ & $0.1078^{5}$ & $0.1108^{6}$ & $0.1026^{2}$ & $0.1076^{4}$ & $0.1004^{1}$ & $0.1059^{3}$ \\
\hline \multirow{3}{*}{$\mathrm{n}$} & Total & $34^{6}$ & $26^{5}$ & $12^{2}$ & $19^{3}$ & $10^{1}$ & $25^{4}$ \\
\hline & Qtd & MLE & MPS & OLS & WLS & $\mathrm{CM}$ & $\mathrm{AD}$ \\
\hline & $\operatorname{Bias}(\theta)$ & $-0.3301^{6}$ & $-0.2589^{3}$ & $-0.1945^{1}$ & $-0.2817^{4}$ & $-0.2243^{2}$ & $-0.3043^{5}$ \\
\hline \multirow{6}{*}{50} & $R M S E(\theta)$ & $0.4431^{6}$ & $0.3578^{2}$ & $0.3511^{1}$ & $0.3849^{4}$ & $0.3644^{3}$ & $0.3947^{5}$ \\
\hline & $\operatorname{Bias}(\lambda)$ & $4.1677^{6}$ & $2.1397^{3}$ & $1.1858^{1}$ & $2.2519^{4}$ & $1.5366^{2}$ & $2.5026^{5}$ \\
\hline & $R M S E(\lambda)$ & $6.7931^{6}$ & $4.4202^{5}$ & $2.8121^{1}$ & $3.7893^{4}$ & $3.0180^{2}$ & $3.7873^{3}$ \\
\hline & $D_{a b s}$ & $0.0684^{3}$ & $0.0700^{6}$ & $0.0663^{2}$ & $0.0693^{5}$ & $0.0650^{1}$ & $0.0688^{4}$ \\
\hline & $D_{\max }$ & $0.0981^{3}$ & $0.0983^{4}$ & $0.0977^{2}$ & $0.1025^{6}$ & $0.0972^{1}$ & $0.1000^{5}$ \\
\hline & Total & $30^{6}$ & $23^{3}$ & $8^{1}$ & $27^{4}$ & $11^{2}$ & $27^{4}$ \\
\hline \multirow[t]{2}{*}{$\mathrm{n}$} & Qtd & MLE & MPS & OLS & WLS & $\mathrm{CM}$ & $\mathrm{AD}$ \\
\hline & $\operatorname{Bias}(\theta)$ & $-0.4012^{4}$ & $-0.3371^{2}$ & $-0.3355^{1}$ & $-0.4015^{5}$ & $-0.3488^{3}$ & $-0.4153^{6}$ \\
\hline \multirow{6}{*}{100} & $\operatorname{RMSE}(\theta)$ & $0.4506^{6}$ & $0.3823^{1}$ & $0.3852^{2}$ & $0.4336^{4}$ & $0.3939^{3}$ & $0.4450^{5}$ \\
\hline & $\operatorname{Bias}(\lambda)$ & $4.8524^{6}$ & $3.0086^{3}$ & $2.5884^{1}$ & $3.7347^{4}$ & $2.7901^{2}$ & $4.0287^{5}$ \\
\hline & $R M S E(\lambda)$ & $6.9720^{6}$ & $4.8351^{4}$ & $3.4891^{1}$ & $4.7115^{3}$ & $3.6423^{2}$ & $4.9055^{5}$ \\
\hline & $D_{a b s}$ & $0.0679^{3}$ & $0.0682^{4}$ & $0.0667^{2}$ & $0.0710^{5}$ & $0.0662^{1}$ & $0.0719^{6}$ \\
\hline & $D_{\max }$ & $0.0974^{2}$ & $0.0962^{1}$ & $0.0990^{3}$ & $0.1055^{6}$ & $0.0990^{4}$ & $0.1048^{5}$ \\
\hline & Total & $27^{4}$ & $15^{2}$ & $10^{1}$ & $27^{4}$ & $15^{2}$ & $32^{6}$ \\
\hline $\mathrm{n}$ & Qtd & MLE & MPS & OLS & WLS & $\mathrm{CM}$ & $\mathrm{AD}$ \\
\hline \multirow{7}{*}{200} & $\operatorname{Bias}(\theta)$ & $-0.4311^{3}$ & $-0.3783^{1}$ & $-0.4310^{2}$ & $-0.4743^{6}$ & $-0.4370^{4}$ & $-0.4649^{5}$ \\
\hline & $R M S E(\theta)$ & $0.4542^{4}$ & $0.4000^{1}$ & $0.4441^{2}$ & $0.4848^{6}$ & $0.4492^{3}$ & $0.4747^{5}$ \\
\hline & $\operatorname{Bias}(\lambda)$ & $4.8749^{5}$ & $3.3545^{1}$ & $4.0376^{2}$ & $5.0660^{6}$ & $4.1746^{3}$ & $4.8398^{4}$ \\
\hline & $R M S E(\lambda)$ & $6.4526^{6}$ & $4.6285^{2}$ & $4.5121^{1}$ & $5.7215^{5}$ & $4.6315^{3}$ & $5.3358^{4}$ \\
\hline & $D_{a b s}$ & $0.0680^{4}$ & $0.0675^{3}$ & $0.0669^{2}$ & $0.0727^{6}$ & $0.0665^{1}$ & $0.0707^{5}$ \\
\hline & $D_{\max }$ & $0.0974^{2}$ & $0.0957^{1}$ & $0.1005^{4}$ & $0.1086^{6}$ & $0.1003^{3}$ & $0.1035^{5}$ \\
\hline & Total & $24^{4}$ & $9^{1}$ & $13^{2}$ & $35^{6}$ & $17^{3}$ & $28^{5}$ \\
\hline
\end{tabular}

Qtd: measures (52)-(55)

The data set was extracted from Lee \& Wang (2003) and refers to remission times (in months) of a randomly censored of 137 bladder cancer patients. Out of 137 data points, 9 observations are right censored. We considered $\left(y_{1}, y_{2}, \ldots, y_{n}\right)$ the observed values from $Y=\min \left(T_{1}, \ldots, T_{M}\right)$. In Table 11 we present, for all models, the maximum likelihood, maximum product of spacings, ordinary least-squares, weighted least-squares, Cramér-von-Mises and Anderson-Darling estimates for $\theta$ and $\lambda$ and its respectivally standard errors estimates. The maximum likelihood estimates were obtained in SAS/SEVERITY procedure (SAS, 2011) and others estimates were 
Table 6 - Simulations results for $\theta=1.0$ and $\lambda=0.5$.

\begin{tabular}{|c|c|c|c|c|c|c|c|}
\hline $\mathrm{n}$ & Qtd & MLE & MPS & OLS & WLS & $\mathrm{CM}$ & $\mathrm{AD}$ \\
\hline \multirow{6}{*}{20} & $\overline{\operatorname{Bias}(\theta)}$ & $-0.2489^{5}$ & $0.1232^{1}$ & $0.2089^{4}$ & $0.2013^{2}$ & $0.2767^{6}$ & $0.2025^{3}$ \\
\hline & $R M S E(\theta)$ & $0.3215^{2}$ & $0.2776^{1}$ & $0.3791^{5}$ & $0.3644^{4}$ & $0.4401^{6}$ & $0.3470^{3}$ \\
\hline & $\operatorname{Bias}(\lambda)$ & $0.8189^{2}$ & $0.7608^{1}$ & $1.0900^{5}$ & $1.0606^{4}$ & $1.3472^{6}$ & $1.0469^{3}$ \\
\hline & $R M S E(\lambda)$ & $1.5344^{3}$ & $1.2123^{1}$ & $1.6737^{5}$ & $1.6190^{4}$ & $1.9892^{6}$ & $1.5311^{2}$ \\
\hline & $D_{a b s}$ & $0.1510^{6}$ & $0.0575^{1}$ & $0.0604^{4}$ & $0.0591^{3}$ & $0.0611^{5}$ & $0.0582^{2}$ \\
\hline & $D_{\max }$ & $0.2645^{6}$ & $0.0894^{1}$ & $0.0994^{4}$ & $0.0970^{3}$ & $0.1053^{5}$ & $0.0958^{2}$ \\
\hline & Total & $24^{4}$ & $6^{1}$ & $27^{5}$ & $20^{3}$ & $34^{6}$ & $15^{2}$ \\
\hline $\mathrm{n}$ & Qtd & MLE & MPS & OLS & WLS & $\mathrm{CM}$ & $\mathrm{AD}$ \\
\hline \multirow{7}{*}{50} & $\operatorname{Bias}(\theta)$ & $-0.2229^{6}$ & $0.0599^{1}$ & $0.1118^{4}$ & $0.1068^{3}$ & $0.1412^{5}$ & $0.1068^{2}$ \\
\hline & $R M S E(\theta)$ & $0.2810^{6}$ & $0.1673^{1}$ & $0.2200^{4}$ & $0.2093^{3}$ & $0.2428^{5}$ & $0.2036^{2}$ \\
\hline & $\operatorname{Bias}(\lambda)$ & $0.5127^{2}$ & $0.3925^{1}$ & $0.5745^{5}$ & $0.5560^{4}$ & $0.6786^{6}$ & $0.5511^{3}$ \\
\hline & $R M S E(\lambda)$ & $1.3774^{6}$ & $0.7404^{1}$ & $0.9340^{4}$ & $0.9037^{3}$ & $1.0337^{5}$ & $0.8914^{2}$ \\
\hline & $D_{a b s}$ & $0.1190^{6}$ & $0.0352^{1}$ & $0.0369^{4}$ & $0.0361^{3}$ & $0.0374^{5}$ & $0.0358^{2}$ \\
\hline & $D_{\max }$ & $0.2046^{6}$ & $0.0557^{1}$ & $0.0610^{4}$ & $0.0594^{3}$ & $0.0634^{5}$ & $0.0589^{2}$ \\
\hline & Total & $32^{6}$ & $6^{1}$ & $25^{4}$ & $19^{3}$ & $31^{5}$ & $13^{2}$ \\
\hline \multirow[t]{2}{*}{$\mathrm{n}$} & Qtd & MLE & MPS & OLS & WLS & $\mathrm{CM}$ & $\mathrm{AD}$ \\
\hline & $\operatorname{Bias}(\theta)$ & $-0.1806^{6}$ & $0.0305^{1}$ & $0.0667^{4}$ & $0.0623^{3}$ & $0.0827^{5}$ & $\overline{0.0621^{2}}$ \\
\hline \multirow{6}{*}{100} & $R M S E(\theta)$ & $0.2226^{6}$ & $0.1175^{1}$ & $0.1514^{4}$ & $0.1422^{3}$ & $0.1624^{5}$ & $0.1394^{2}$ \\
\hline & $\operatorname{Bias}(\lambda)$ & $0.1451^{1}$ & $0.2106^{2}$ & $0.3412^{5}$ & $0.3244^{4}$ & $0.3990^{6}$ & $0.3207^{3}$ \\
\hline & $R M S E(\lambda)$ & $0.9812^{6}$ & $0.5223^{1}$ & $0.6462^{4}$ & $0.6216^{3}$ & $0.6946^{5}$ & $0.6140^{2}$ \\
\hline & $D_{a b s}$ & $0.0798^{6}$ & $0.0243^{1}$ & $0.0257^{4}$ & $0.0250^{3}$ & $0.0259^{5}$ & $0.0249^{2}$ \\
\hline & $D_{\max }$ & $0.1335^{6}$ & $0.0390^{1}$ & $0.0425^{4}$ & $0.0412^{3}$ & $0.0436^{5}$ & $0.0409^{2}$ \\
\hline & Total & $31^{5}$ & $7^{1}$ & $25^{4}$ & $19^{3}$ & $31^{5}$ & $13^{2}$ \\
\hline $\mathrm{n}$ & Qtd & MLE & MPS & OLS & WLS & $\mathrm{CM}$ & $\mathrm{AD}$ \\
\hline \multirow{7}{*}{200} & $\operatorname{Bias}(\theta)$ & $-0.1434^{6}$ & $0.0115^{1}$ & $0.0360^{4}$ & $0.0328^{3}$ & $0.0450^{5}$ & $0.0324^{2}$ \\
\hline & $R M S E(\theta)$ & $0.1642^{6}$ & $0.0848^{1}$ & $0.1064^{4}$ & $0.0988^{3}$ & $0.1115^{5}$ & $0.0974^{2}$ \\
\hline & $\operatorname{Bias}(\lambda)$ & $-0.1500^{2}$ & $0.0900^{1}$ & $0.1836^{5}$ & $0.1708^{4}$ & $0.2172^{6}$ & $0.1670^{3}$ \\
\hline & $R M S E(\lambda)$ & $0.5455^{6}$ & $0.3820^{1}$ & $0.4597^{4}$ & $0.4397^{3}$ & $0.4827^{5}$ & $0.4351^{2}$ \\
\hline & $D_{a b s}$ & $0.0463^{6}$ & $0.0170^{1}$ & $0.0180^{4}$ & $0.0175^{3}$ & $0.0181^{5}$ & $0.0174^{2}$ \\
\hline & $D_{\max }$ & $0.0757^{6}$ & $0.0276^{1}$ & $0.0298^{4}$ & $0.0289^{3}$ & $0.0303^{5}$ & $0.0288^{2}$ \\
\hline & Total & $32^{6}$ & $6^{1}$ & $25^{4}$ & $19^{3}$ & $31^{5}$ & $13^{2}$ \\
\hline
\end{tabular}

Qtd: measures (52)-(55)

obtained in $R$ version 2.15, using the "fitdist", "max.Lik" and " $n l s$ " functions. The dotted in Table 11 indicates is not possible to calculate standard errors estimates to the Cramér-von-Mises and Anderson-Darling methods.

From Table 11, it is observed that all estimation methods were effective to estimate the parameters $\theta$ and $\lambda$, in addition, the standard errors there were small. 
Table 7 - Simulations results for $\theta=1.0$ and $\lambda=1.0$.

\begin{tabular}{|c|c|c|c|c|c|c|c|}
\hline $\mathrm{n}$ & Qtd & MLE & MPS & OLS & WLS & $\mathrm{CM}$ & $\mathrm{AD}$ \\
\hline \multirow{7}{*}{20} & $\overline{\operatorname{Bias}(\theta)}$ & $-0.3030^{6}$ & $0.0523^{1}$ & $0.1225^{3}$ & $0.1195^{2}$ & $0.1905^{5}$ & $0.1284^{4}$ \\
\hline & $\operatorname{RMSE}(\theta)$ & $0.3587^{5}$ & $0.2317^{1}$ & $0.3077^{4}$ & $0.2964^{3}$ & $0.3603^{6}$ & $0.2860^{2}$ \\
\hline & $\operatorname{Bias}(\lambda)$ & $0.3035^{1}$ & $0.4394^{2}$ & $0.7778^{5}$ & $0.7559^{3}$ & $1.0902^{6}$ & $0.7692^{4}$ \\
\hline & $R M S E(\lambda)$ & $1.4500^{3}$ & $1.1249^{1}$ & $1.5984^{5}$ & $1.5354^{4}$ & $1.9588^{6}$ & $1.4486^{2}$ \\
\hline & $D_{a b s}$ & $0.1419^{6}$ & $0.0556^{1}$ & $0.0585^{4}$ & $0.0571^{3}$ & $0.0596^{5}$ & $0.0567^{2}$ \\
\hline & $D_{\max }$ & $0.2588^{6}$ & $0.0863^{1}$ & $0.0953^{4}$ & $0.0931^{3}$ & $0.1016^{5}$ & $0.0927^{2}$ \\
\hline & Total & $27^{5}$ & $7^{1}$ & $25^{4}$ & $18^{3}$ & $33^{6}$ & $16^{2}$ \\
\hline \multirow[t]{2}{*}{$\mathrm{n}$} & Qtd & MLE & MPS & OLS & WLS & $\mathrm{CM}$ & $\mathrm{AD}$ \\
\hline & $\operatorname{Bias}(\theta)$ & $-0.2720^{6}$ & $0.0059^{1}$ & $0.0481^{2}$ & $0.0484^{3}$ & $0.0789^{5}$ & $0.0520^{4}$ \\
\hline \multirow{6}{*}{50} & $R M S E(\theta)$ & $0.3156^{6}$ & $0.1479^{1}$ & $0.1820^{4}$ & $0.1740^{3}$ & $0.1990^{5}$ & $0.1707^{2}$ \\
\hline & $\operatorname{Bias}(\lambda)$ & $-0.0562^{1}$ & $0.1132^{2}$ & $0.2975^{4}$ & $0.2934^{3}$ & $0.4289^{6}$ & $0.3039^{5}$ \\
\hline & $R M S E(\lambda)$ & $1.3670^{6}$ & $0.7181^{1}$ & $0.8698^{4}$ & $0.8449^{3}$ & $0.9642^{5}$ & $0.8401^{2}$ \\
\hline & $D_{a b s}$ & $0.1039^{6}$ & $0.0344^{1}$ & $0.0361^{4}$ & $0.0353^{3}$ & $0.0367^{5}$ & $0.0352^{2}$ \\
\hline & $D_{\max }$ & $0.1900^{6}$ & $0.0552^{1}$ & $0.0597^{4}$ & $0.0583^{3}$ & $0.0620^{5}$ & $0.0581^{2}$ \\
\hline & Total & $31^{5}$ & $7^{1}$ & $22^{4}$ & $18^{3}$ & $31^{5}$ & $17^{2}$ \\
\hline \multirow[t]{4}{*}{$\mathrm{n}$} & Qtd & MLE & MPS & OLS & WLS & $\mathrm{CM}$ & $\mathrm{AD}$ \\
\hline & $\operatorname{Bias}(\theta)$ & $-0.2281^{6}$ & $-0.0107^{1}$ & $0.0178^{2}$ & $0.0195^{3}$ & $0.0355^{5}$ & $0.0209^{4}$ \\
\hline & $R M S E(\theta)$ & $0.2544^{6}$ & $0.1121^{1}$ & $0.1312^{4}$ & $0.1240^{3}$ & $0.1376^{5}$ & $0.1223^{2}$ \\
\hline & $\operatorname{Bias}(\lambda)$ & $-0.4776^{6}$ & $-0.0162^{1}$ & $0.1119^{2}$ & $0.1161^{3}$ & $0.1896^{5}$ & $0.1200^{4}$ \\
\hline \multirow[t]{4}{*}{100} & $R M S E(\lambda)$ & $1.0505^{6}$ & $0.5602^{1}$ & $0.6337^{4}$ & $0.6144^{3}$ & $0.6690^{5}$ & $0.6103^{2}$ \\
\hline & $D_{a b s}$ & $0.0623^{6}$ & $0.0245^{1}$ & $0.0256^{4}$ & $0.0251^{3}$ & $0.0259^{5}$ & $0.0250^{2}$ \\
\hline & $D_{\max }$ & $0.1132^{6}$ & $0.0401^{1}$ & $0.0427^{4}$ & $0.0417^{3}$ & $0.0436^{5}$ & $0.0416^{2}$ \\
\hline & Total & $36^{6}$ & $6^{1}$ & $20^{4}$ & $18^{3}$ & $30^{5}$ & $16^{2}$ \\
\hline \multirow[t]{4}{*}{$\mathrm{n}$} & Qtd & MLE & MPS & OLS & WLS & $\mathrm{CM}$ & $\mathrm{AD}$ \\
\hline & $\operatorname{Bias}(\theta)$ & $-0.1964^{6}$ & $-0.0158^{5}$ & $0.0019^{1}$ & $0.0049^{2}$ & $0.0123^{4}$ & $0.0051^{3}$ \\
\hline & $\operatorname{RMSE}(\theta)$ & $0.2061^{6}$ & $0.0865^{1}$ & $0.0983^{4}$ & $0.0913^{3}$ & $0.1000^{5}$ & $0.0906^{2}$ \\
\hline & $\operatorname{Bias}(\lambda)$ & $-0.7545^{6}$ & $-0.0663^{5}$ & $0.0164^{1}$ & $0.0281^{3}$ & $0.0633^{4}$ & $0.0278^{2}$ \\
\hline \multirow[t]{4}{*}{200} & $R M S E(\lambda)$ & $0.8698^{6}$ & $0.4478^{1}$ & $0.4863^{4}$ & $0.4654^{3}$ & $0.4956^{5}$ & $0.4632^{2}$ \\
\hline & $D_{a b s}$ & $0.0340^{6}$ & $0.0178^{1}$ & $0.0184^{4}$ & $0.0180^{3}$ & $0.0185^{5}$ & $0.0180^{2}$ \\
\hline & $D_{\max }$ & $0.0632^{6}$ & $0.0294^{1}$ & $0.0310^{4}$ & $0.0301^{3}$ & $0.0313^{5}$ & $0.0301^{2}$ \\
\hline & Total & $36^{6}$ & $14^{2}$ & $18^{4}$ & $17^{3}$ & $28^{5}$ & $13^{1}$ \\
\hline
\end{tabular}

Qtd: measures (52)-(55)

The SAS/SEVERITY procedure can fit multiple distributions at the same time and choose the best distribution according to a specified selection criterion. Seven different statistics of fit can be used as selection criteria. They are log likelihood, Akaike's information criterion (AIC), corrected Akaike's information criterion (AICC), Schwarz Bayesian information criterion (BIC), Kolmogorov-Smirnov statistic (KS), Anderson-Darling statistic (AD) and Cramér-von-Mises statistic $(\mathrm{CvM})$. The calculed values of theses statistics are report in Table 12. In Figure 6 is possible to see similar fit for the five models applied to the data set. 
Table 8 - Simulations results for $\theta=1.0$ and $\lambda=2.0$.

\begin{tabular}{|c|c|c|c|c|c|c|c|}
\hline $\mathrm{n}$ & Qtd & MLE & MPS & OLS & WLS & $\mathrm{CM}$ & $\mathrm{AD}$ \\
\hline \multirow{6}{*}{20} & $\overline{\operatorname{Bias}(\theta)}$ & $-0.5561^{6}$ & $-0.0306^{3}$ & $0.0246^{1}$ & $0.0305^{2}$ & $0.0950^{5}$ & $0.0450^{4}$ \\
\hline & $R M S E(\theta)$ & $0.6078^{6}$ & $0.2018^{1}$ & $0.2452^{4}$ & $0.2374^{3}$ & $0.2801^{5}$ & $0.2278^{2}$ \\
\hline & $\operatorname{Bias}(\lambda)$ & $2.6529^{6}$ & $-0.0483^{1}$ & $0.3488^{2}$ & $0.3745^{3}$ & $0.8225^{5}$ & $0.4129^{4}$ \\
\hline & $R M S E(\lambda)$ & $5.7000^{6}$ & $1.2598^{1}$ & $1.8160^{4}$ & $1.7827^{3}$ & $2.2937^{5}$ & $1.5761^{2}$ \\
\hline & $D_{a b s}$ & $0.2666^{6}$ & $0.0553^{1}$ & $0.0576^{4}$ & $0.0562^{2}$ & $0.0589^{5}$ & $0.0562^{3}$ \\
\hline & $D_{\max }$ & $0.5405^{6}$ & $0.0872^{1}$ & $0.0940^{4}$ & $0.0919^{2}$ & $0.0998^{5}$ & $0.0921^{3}$ \\
\hline & Total & $36^{6}$ & $8^{1}$ & $19^{4}$ & $15^{2}$ & $30^{5}$ & $18^{3}$ \\
\hline $\mathrm{n}$ & Qtd & MLE & MPS & OLS & WLS & $\mathrm{CM}$ & $\mathrm{AD}$ \\
\hline \multirow{7}{*}{50} & $\operatorname{Bias}(\theta)$ & $-0.5441^{6}$ & $-0.0400^{5}$ & $-0.0070^{2}$ & $0.0015^{1}$ & $0.0261^{4}$ & $0.0074^{3}$ \\
\hline & $R M S E(\theta)$ & $0.5956^{6}$ & $0.1429^{1}$ & $0.1583^{4}$ & $0.1496^{3}$ & $0.1642^{5}$ & $0.1465^{2}$ \\
\hline & $\operatorname{Bias}(\lambda)$ & $2.4653^{6}$ & $-0.1947^{4}$ & $0.0087^{1}$ & $0.0502^{2}$ & $0.2088^{5}$ & $0.0806^{3}$ \\
\hline & $R M S E(\lambda)$ & $5.5220^{6}$ & $0.8887^{1}$ & $0.9991^{4}$ & $0.9519^{3}$ & $1.0661^{5}$ & $0.9413^{2}$ \\
\hline & $D_{a b s}$ & $0.2397^{6}$ & $0.0360^{1}$ & $0.0372^{4}$ & $0.0363^{3}$ & $0.0376^{5}$ & $0.0363^{2}$ \\
\hline & $D_{\max }$ & $0.5110^{6}$ & $0.0588^{1}$ & $0.0619^{4}$ & $0.0603^{3}$ & $0.0633^{5}$ & $0.0603^{2}$ \\
\hline & Total & $36^{6}$ & $13^{1}$ & $19^{4}$ & $15^{3}$ & $29^{5}$ & $14^{2}$ \\
\hline $\mathrm{n}$ & Qtd & MLE & MPS & OLS & WLS & $\mathrm{CM}$ & $\mathrm{AD}$ \\
\hline \multirow{7}{*}{100} & $\operatorname{Bias}(\theta)$ & $-0.6681^{6}$ & $-0.0297^{5}$ & $-0.0087^{3}$ & $-0.0012^{2}$ & $0.0094^{4}$ & $0.0007^{1}$ \\
\hline & $R M S E(\theta)$ & $0.7091^{6}$ & $0.1062^{2}$ & $0.1157^{4}$ & $0.1072^{3}$ & $0.1164^{5}$ & $0.1057^{1}$ \\
\hline & $\operatorname{Bias}(\lambda)$ & $5.7622^{6}$ & $-0.1576^{5}$ & $-0.0328^{3}$ & $0.0077^{1}$ & $0.0757^{4}$ & $0.0181^{2}$ \\
\hline & $R M S E(\lambda)$ & $8.0531^{6}$ & $0.6714^{1}$ & $0.7238^{4}$ & $0.6809^{3}$ & $0.7354^{5}$ & $0.6744^{2}$ \\
\hline & $D_{a b s}$ & $0.3302^{6}$ & $0.0260^{1}$ & $0.0268^{4}$ & $0.0260^{3}$ & $0.0268^{5}$ & $0.0260^{2}$ \\
\hline & $D_{\max }$ & $0.7144^{6}$ & $0.0429^{1}$ & $0.0448^{4}$ & $0.0434^{3}$ & $0.0452^{5}$ & $0.0433^{2}$ \\
\hline & Total & $36^{6}$ & $15^{2}$ & $22^{4}$ & $15^{2}$ & $28^{5}$ & $10^{1}$ \\
\hline $\mathrm{n}$ & Qtd & MLE & MPS & OLS & WLS & $\mathrm{CM}$ & $\mathrm{AD}$ \\
\hline \multirow{7}{*}{200} & $\operatorname{Bias}(\theta)$ & $-0.7871^{6}$ & $-0.0176^{5}$ & $-0.0051^{4}$ & $-0.0002^{2}$ & $0.0041^{3}$ & $0.0000^{1}$ \\
\hline & $R M S E(\theta)$ & $0.8030^{6}$ & $0.0745^{1}$ & $0.0819^{5}$ & $0.0752^{3}$ & $0.0818^{4}$ & $0.0747^{2}$ \\
\hline & $\operatorname{Bias}(\lambda)$ & $9.1652^{6}$ & $-0.0938^{5}$ & $-0.0209^{3}$ & $0.0063^{1}$ & $0.0338^{4}$ & $0.0070^{2}$ \\
\hline & $R M S E(\lambda)$ & $10.2663^{6}$ & $0.4729^{1}$ & $0.5093^{4}$ & $0.4757^{3}$ & $0.5115^{5}$ & $0.4734^{2}$ \\
\hline & $D_{a b s}$ & $0.4214^{6}$ & $0.0184^{1}$ & $0.0190^{5}$ & $0.0184^{3}$ & $0.0190^{4}$ & $0.0184^{2}$ \\
\hline & $D_{\max }$ & $0.9089^{6}$ & $0.0305^{1}$ & $0.0318^{4}$ & $0.0307^{3}$ & $0.0319^{5}$ & $0.0307^{2}$ \\
\hline & $\overline{\text { Total }}$ & $36^{6}$ & $14^{2}$ & $25^{4}$ & $15^{3}$ & $25^{4}$ & $11^{1}$ \\
\hline
\end{tabular}

Qtd: measures (52)-(55)

A close examination of Table 12 reveals that the zero truncated Lindley-Poisson model is the best choice among the competing models, since it has the lowest AIC, AICC and others statistics. This is also supported by the survival curves in Figure 6. 
Table 9 - Simulations results for $\theta=1.0$ and $\lambda=3.0$.

\begin{tabular}{|c|c|c|c|c|c|c|c|}
\hline $\mathrm{n}$ & Qtd & MLE & MPS & OLS & WLS & $\mathrm{CM}$ & $\mathrm{AD}$ \\
\hline \multirow{6}{*}{20} & $\overline{\operatorname{Bias}(\theta)}$ & $-0.8247^{6}$ & $-0.0589^{4}$ & $-0.0104^{2}$ & $0.0024^{1}$ & $0.0624^{5}$ & $0.0179^{3}$ \\
\hline & $R M S E(\theta)$ & $0.8338^{6}$ & $0.1934^{1}$ & $0.2261^{4}$ & $0.2156^{3}$ & $0.2494^{5}$ & $0.2045^{2}$ \\
\hline & $\operatorname{Bias}(\lambda)$ & $9.2201^{6}$ & $-0.3015^{3}$ & $0.1933^{1}$ & $0.2768^{2}$ & $0.8875^{5}$ & $0.3340^{4}$ \\
\hline & $R M S E(\lambda)$ & $11.0600^{6}$ & $1.5584^{1}$ & $2.3313^{3}$ & $2.3513^{4}$ & $3.0018^{5}$ & $1.9532^{2}$ \\
\hline & $D_{a b s}$ & $0.4286^{6}$ & $0.0572^{1}$ & $0.0591^{4}$ & $0.0577^{3}$ & $0.0598^{5}$ & $0.0574^{2}$ \\
\hline & $D_{\max }$ & $0.9199^{6}$ & $0.0907^{1}$ & $0.0965^{4}$ & $0.0942^{3}$ & $0.1010^{5}$ & $0.0937^{2}$ \\
\hline & Total & $36^{6}$ & $11^{1}$ & $18^{4}$ & $16^{3}$ & $30^{5}$ & $15^{2}$ \\
\hline $\mathrm{n}$ & Qtd & MLE & MPS & OLS & WLS & $\mathrm{CM}$ & $\mathrm{AD}$ \\
\hline \multirow{7}{*}{50} & $\operatorname{Bias}(\theta)$ & $-0.8720^{6}$ & $-0.0426^{5}$ & $-0.0125^{3}$ & $-0.0027^{1}$ & $0.0188^{4}$ & $0.0030^{2}$ \\
\hline & $R M S E(\theta)$ & $0.8739^{6}$ & $0.1292^{2}$ & $0.1424^{4}$ & $0.1323^{3}$ & $0.1452^{5}$ & $0.1284^{1}$ \\
\hline & $\operatorname{Bias}(\lambda)$ & $11.4698^{6}$ & $-0.2674^{5}$ & $-0.0195^{1}$ & $0.0435^{2}$ & $0.2370^{4}$ & $0.0825^{3}$ \\
\hline & $R M S E(\lambda)$ & $12.4036^{6}$ & $0.9986^{1}$ & $1.1754^{4}$ & $1.0898^{3}$ & $1.2636^{5}$ & $1.0599^{2}$ \\
\hline & $D_{a b s}$ & $0.4824^{6}$ & $0.0368^{2}$ & $0.0380^{4}$ & $0.0368^{3}$ & $0.0381^{5}$ & $0.0367^{1}$ \\
\hline & $D_{\max }$ & $0.9852^{6}$ & $0.0599^{1}$ & $0.0630^{4}$ & $0.0608^{3}$ & $0.0638^{5}$ & $0.0605^{2}$ \\
\hline & Total & $36^{6}$ & $16^{3}$ & $20^{4}$ & $15^{2}$ & $28^{5}$ & $11^{1}$ \\
\hline \multirow[t]{2}{*}{$\mathrm{n}$} & Qtd & MLE & MPS & OLS & WLS & $\mathrm{CM}$ & $\mathrm{AD}$ \\
\hline & $\overline{\operatorname{Bias}(\theta)}$ & $-0.8932^{6}$ & $-0.0256^{5}$ & $-0.0067^{3}$ & $-0.0002^{1}$ & $0.0089^{4}$ & $0.0011^{2}$ \\
\hline \multirow{6}{*}{100} & $R M S E(\theta)$ & $0.8934^{6}$ & $0.0905^{2}$ & $0.0990^{4}$ & $0.0914^{3}$ & $0.0997^{5}$ & $0.0901^{1}$ \\
\hline & $\operatorname{Bias}(\alpha)$ & $14.3461^{6}$ & $-0.1629^{5}$ & $-0.0153^{1}$ & $0.0270^{2}$ & $0.1082^{4}$ & $0.0371^{3}$ \\
\hline & $R M S E(\alpha)$ & $15.0065^{6}$ & $0.7038^{1}$ & $0.7908^{4}$ & $0.7334^{3}$ & $0.8150^{5}$ & $0.7240^{2}$ \\
\hline & $D_{a b s}$ & $0.5000^{6}$ & $0.0260^{2}$ & $0.0269^{5}$ & $0.0260^{3}$ & $0.0269^{4}$ & $0.0260^{1}$ \\
\hline & $D_{\max }$ & $0.9985^{6}$ & $0.0426^{1}$ & $0.0447^{4}$ & $0.0431^{3}$ & $0.0450^{5}$ & $0.0429^{2}$ \\
\hline & Total & $36^{6}$ & $16^{3}$ & $21^{4}$ & $15^{2}$ & $27^{5}$ & $11^{1}$ \\
\hline $\mathrm{n}$ & Qtd & MLE & MPS & OLS & WLS & $\mathrm{CM}$ & $\mathrm{AD}$ \\
\hline \multirow{7}{*}{200} & $\operatorname{Bias}(\theta)$ & $-0.9042^{6}$ & $-0.0140^{5}$ & $-0.0032^{3}$ & $0.0007^{2}$ & $0.0045^{4}$ & $0.0007^{1}$ \\
\hline & $R M S E(\theta)$ & $0.9042^{6}$ & $0.0627^{1}$ & $0.0693^{4}$ & $0.0638^{3}$ & $0.0696^{5}$ & $0.0634^{2}$ \\
\hline & $\operatorname{Bias}(\lambda)$ & $17.4592^{6}$ & $-0.0880^{5}$ & $-0.0073^{1}$ & $0.0186^{2}$ & $0.0532^{4}$ & $0.0190^{3}$ \\
\hline & $R M S E(\lambda)$ & $17.9389^{6}$ & $0.4913^{1}$ & $0.5473^{4}$ & $0.5074^{3}$ & $0.5553^{5}$ & $0.5040^{2}$ \\
\hline & $D_{a b s}$ & $0.5016^{6}$ & $0.0183^{1}$ & $0.0190^{5}$ & $0.0184^{3}$ & $0.0190^{4}$ & $0.0184^{2}$ \\
\hline & $D_{\max }$ & $0.9997^{6}$ & $0.0301^{1}$ & $0.0317^{4}$ & $0.0305^{3}$ & $0.0318^{5}$ & $0.0304^{2}$ \\
\hline & Total & $36^{6}$ & $14^{2}$ & $21^{4}$ & $16^{3}$ & $27^{5}$ & $12^{1}$ \\
\hline
\end{tabular}

Qtd: measures (52)-(55)

\section{CONCLUDING REMARKS}

In this paper we proposed the composed zero truncated Lindley-Poisson distribution, which was obtained by compounding an one parameter Lindley distribution with a zero truncated Poisson under the first and last failure time when a device is subjected to the presence of an unknown number $M$ of causes of failures. Both alternative distributions have the one parameter Lindley distribution as a particular case. For the first distribution we assume we have a series system and observe the time to the first failure, $Y=\min \left(T_{1}, \ldots, T_{M}\right)$ while for the second distribution 
Table $10-$ Simulations results for $\theta=1.0$ and $\lambda=5.0$.

\begin{tabular}{|c|c|c|c|c|c|c|c|}
\hline $\mathrm{n}$ & Qtd & MLE & MPS & OLS & WLS & $\mathrm{CM}$ & $\mathrm{AD}$ \\
\hline \multirow{6}{*}{20} & $\overline{\operatorname{Bias}(\theta)}$ & $-0.8996^{6}$ & $-0.0615^{5}$ & $-0.0154^{3}$ & $-0.0030^{1}$ & $0.0543^{4}$ & $0.0123^{2}$ \\
\hline & $R M S E(\theta)$ & $0.9001^{6}$ & $0.1756^{1}$ & $0.2061^{4}$ & $0.1973^{3}$ & $0.2240^{5}$ & $0.1826^{2}$ \\
\hline & $\operatorname{Bias}(\lambda)$ & $12.0483^{6}$ & $-0.4518^{2}$ & $0.4199^{1}$ & $0.5822^{3}$ & $1.6382^{5}$ & $0.6302^{4}$ \\
\hline & $R M S E(\lambda)$ & $13.6893^{6}$ & $2.4622^{1}$ & $3.8755^{3}$ & $4.4187^{4}$ & $5.1322^{5}$ & $3.3243^{2}$ \\
\hline & $D_{a b s}$ & $0.4572^{6}$ & $0.0586^{2}$ & $0.0607^{5}$ & $0.0591^{3}$ & $0.0605^{4}$ & $0.0579^{1}$ \\
\hline & $D_{\max }$ & $0.9920^{6}$ & $0.0925^{1}$ & $0.0984^{4}$ & $0.0958^{3}$ & $0.1011^{5}$ & $0.0936^{2}$ \\
\hline & Total & $36^{6}$ & $12^{1}$ & $20^{4}$ & $17^{3}$ & $28^{5}$ & $13^{2}$ \\
\hline $\mathrm{n}$ & Qtd & MLE & MPS & OLS & WLS & $\mathrm{CM}$ & $\mathrm{AD}$ \\
\hline \multirow{7}{*}{50} & $\operatorname{Bias}(\theta)$ & $-0.9176^{6}$ & $-0.0380^{5}$ & $-0.0070^{3}$ & $0.0007^{1}$ & $0.0203^{4}$ & $0.0038^{2}$ \\
\hline & $R M S E(\theta)$ & $0.9178^{6}$ & $0.1085^{1}$ & $0.1240^{4}$ & $0.1142^{3}$ & $0.1286^{5}$ & $0.1100^{2}$ \\
\hline & $\operatorname{Bias}(\lambda)$ & $17.1703^{6}$ & $-0.3598^{4}$ & $0.0956^{1}$ & $0.1594^{2}$ & $0.4949^{5}$ & $0.1863^{3}$ \\
\hline & $R M S E(\lambda)$ & $18.4769^{6}$ & $1.2917^{1}$ & $1.7876^{4}$ & $1.6126^{3}$ & $2.0372^{5}$ & $1.5073^{2}$ \\
\hline & $D_{a b s}$ & $0.4771^{6}$ & $0.0367^{2}$ & $0.0383^{5}$ & $0.0369^{3}$ & $0.0382^{4}$ & $0.0366^{1}$ \\
\hline & $D_{\max }$ & $0.9992^{6}$ & $0.0589^{1}$ & $0.0628^{4}$ & $0.0602^{3}$ & $0.0635^{5}$ & $0.0595^{2}$ \\
\hline & Total & $36^{6}$ & $14^{2}$ & $21^{4}$ & $15^{3}$ & $28^{5}$ & $12^{1}$ \\
\hline \multirow[t]{2}{*}{$\mathrm{n}$} & Qtd & MLE & MPS & OLS & WLS & $\mathrm{CM}$ & $\mathrm{AD}$ \\
\hline & $\operatorname{Bias}(\theta)$ & $-0.9280^{6}$ & $-0.0228^{5}$ & $-0.0040^{3}$ & $0.0011^{1}$ & $0.0094^{4}$ & $0.0015^{2}$ \\
\hline \multirow{6}{*}{100} & $R M S E(\theta)$ & $0.9280^{6}$ & $0.0750^{1}$ & $0.0858^{4}$ & $0.0782^{3}$ & $0.0872^{5}$ & $0.0767^{2}$ \\
\hline & $\operatorname{Bias}(\lambda)$ & $22.5263^{6}$ & $-0.2274^{5}$ & $0.0285^{1}$ & $0.0759^{2}$ & $0.2131^{4}$ & $0.0803^{3}$ \\
\hline & $R M S E(\lambda)$ & $23.4630^{6}$ & $0.8913^{1}$ & $1.1314^{4}$ & $1.0183^{3}$ & $1.2009^{5}$ & $0.9893^{2}$ \\
\hline & $D_{a b s}$ & $0.4781^{6}$ & $0.0257^{1}$ & $0.0270^{5}$ & $0.0260^{3}$ & $0.0270^{4}$ & $0.0259^{2}$ \\
\hline & $D_{\max }$ & $0.9999^{6}$ & $0.0415^{1}$ & $0.0444^{4}$ & $0.0424^{3}$ & $0.0447^{5}$ & $0.0421^{2}$ \\
\hline & Total & $36^{6}$ & $14^{2}$ & $21^{4}$ & $15^{3}$ & $27^{5}$ & $13^{1}$ \\
\hline $\mathrm{n}$ & Qtd & MLE & MPS & OLS & WLS & $\mathrm{CM}$ & $\mathrm{AD}$ \\
\hline \multirow{7}{*}{200} & $\operatorname{Bias}(\theta)$ & $-0.9396^{6}$ & $-0.0131^{5}$ & $-0.0022^{3}$ & $0.0008^{2}$ & $0.0044^{4}$ & $0.0005^{1}$ \\
\hline & $R M S E(\theta)$ & $0.9396^{6}$ & $0.0523^{1}$ & $0.0600^{4}$ & $0.0544^{3}$ & $0.0604^{5}$ & $0.0539^{2}$ \\
\hline & $\operatorname{Bias}(\lambda)$ & $31.1841^{6}$ & $-0.1367^{5}$ & $0.0051^{1}$ & $0.0364^{3}$ & $0.0943^{4}$ & $0.0331^{2}$ \\
\hline & $R M S E(\lambda)$ & $31.3809^{6}$ & $0.6269^{1}$ & $0.7660^{4}$ & $0.6866^{3}$ & $0.7875^{5}$ & $0.6766^{2}$ \\
\hline & $D_{a b s}$ & $0.4862^{6}$ & $0.0181^{1}$ & $0.0191^{5}$ & $0.0183^{3}$ & $0.0191^{4}$ & $0.0183^{2}$ \\
\hline & $D_{\max }$ & $1.0000^{6}$ & $0.0293^{1}$ & $0.0314^{4}$ & $0.0299^{3}$ & $0.0315^{5}$ & $0.0298^{2}$ \\
\hline & Total & $36^{6}$ & $14^{2}$ & $21^{4}$ & $17^{3}$ & $27^{5}$ & $11^{1}$ \\
\hline
\end{tabular}

Qtd: measures (52)-(55)

we assume we have a parallel system and observe the time to the last failure of the device, $Y=\max \left(T_{1}, \ldots, T_{M}\right)$.

We compared, via intensive simulation experiments, the estimation of parameters of the zero truncated Lindley-Poisson distribution using six known estimation methods, namely: the maximum likelihood, maximum product of spacings, ordinal and weighted least-squares, Cramér-von Mises and Anderson-Darling. 


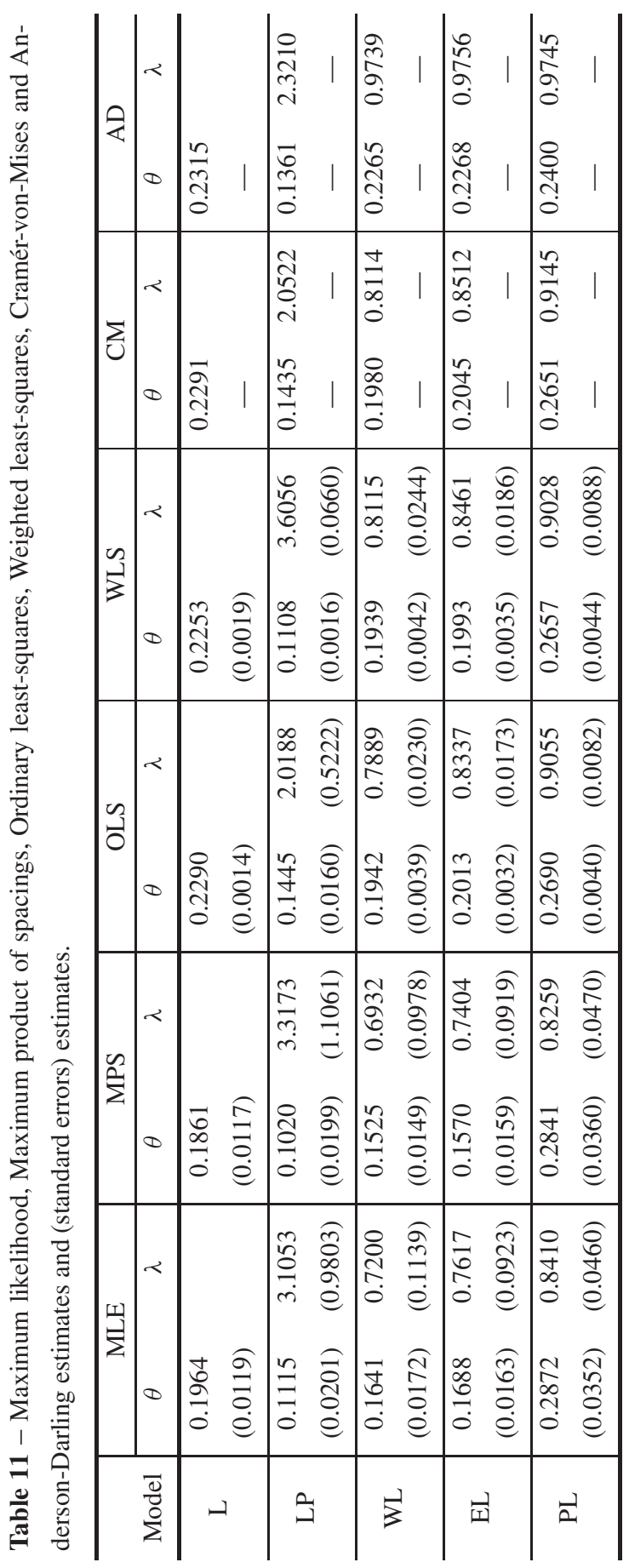


Table 12 - -2log-likelihood values and goodness of fit measures.

\begin{tabular}{llllllll}
\hline Model & $-2 \times \log l i k$ & AIC & AICC & BIC & KS & AD & CvM \\
\hline L & 895.7115 & 897.7115 & 897.7411 & 900.6314 & 1.4358 & 3.0061 & 0.5537 \\
LP & 879.3024 & 883.3024 & 883.3920 & 889.1424 & 1.3375 & 332.3061 & 0.5713 \\
WL & 890.9094 & 894.9094 & 894.9990 & 900.7494 & 11.7211 & 84085.45 & 59.2112 \\
EL & 890.4974 & 894.4774 & 894.5669 & 900.3173 & 1.1176 & 1.5698 & 0.02796 \\
PL & 884.2719 & 888.2719 & 888.3615 & 894.1119 & 0.8391 & 0.9585 & 0.1384 \\
\hline
\end{tabular}

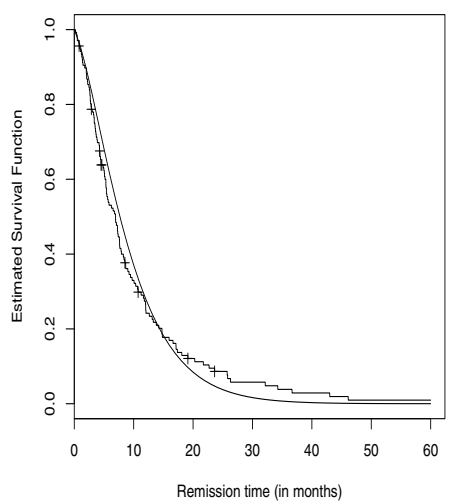

(a) $\mathrm{L}$

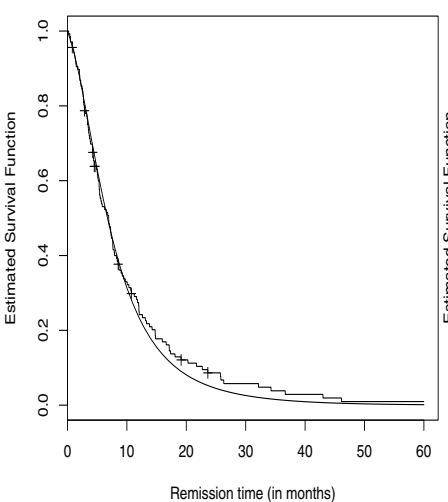

(b) LP

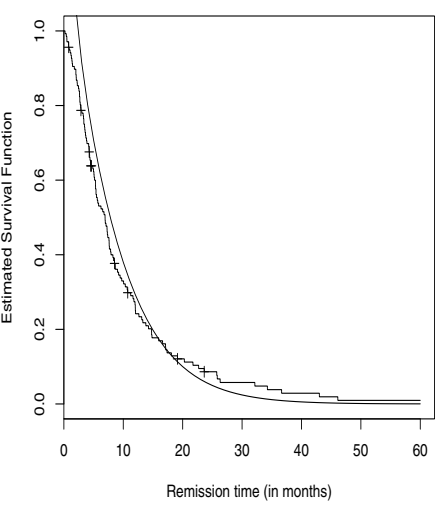

(c) WL

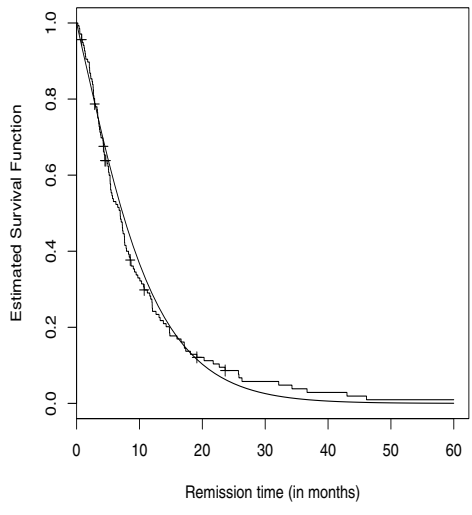

(d) EL

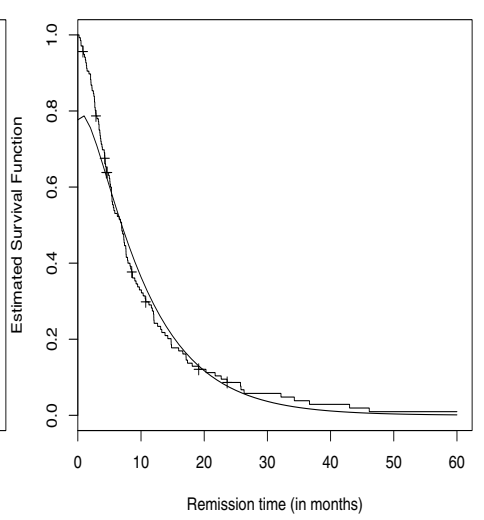

(e) PL

Figure 6 - Fitted survival curves.

In general, the methods of estimation showed to be efficient to estimate the parameters of the zero truncated Lindley-Poisson distribution. Motivated by application in real data set, we hope this model may be able to attract wider applicability in survival and reliability. For possible future works, there the interest of authors in studies of the Fisher information matrix, Confidence intervals, Hypothesis test and Bayesian estimates. 


\section{REFERENCES}

[1] ALI S. 2015. On the bayesian estimation of the weighted lindley distribution. Journal of Statistical Computation and Simulation, 85(5): 855-880.

[2] Arnold BC, Balakrishnan N \& Nagaraja HN. 2008. A first course in order statistics. Vol. 54 of Classics in Applied Mathematics. Society for Industrial and Applied Mathematics (SIAM), Philadelphia, PA.

[3] Bakouch HS, Al-Zahrani BM, Al-Shomrani AA, Marchi, Vitor AA \& LouzadaNeto F. 2012. An extended Lindley distribution. Journal of the Korean Statistical Society, 41(1): $75-85$.

[4] Barreto-Souza W \& Bakouch HS. 2013. A new lifetime model with decreasing failure rate. Statistics: A Journal of Theoretical and Applied Statistics, 47(2): 465-476.

[5] BASU AP. 1981. Identifiability problems in the theory of competing and complementary risks - a survey. In: Statistical distributions in scientific work, Vol. 5 (Trieste, 1980). Vol. 79 of NATO Adv. Study Inst. Ser. C: Math. Phys. Sci. Reidel, Dordrecht, pp. 335-347.

[6] Borah M \& Begum RA. 2002. Some properties of Poisson-Lindley and its derived distributions. Journal of the Indian Statistical Association, 40(1): 13-25.

[7] Borah M \& Deka NA. 2001. Poisson-Lindley and some of its mixture distributions. Pure and Applied Mathematika Sciences, 53(1-2): 1-8.

[8] Borah M \& DeKa NA. 2001. A study on the inflated Poisson Lindley distribution. Journal of the Indian Society of Agricultural Statistics, 54(3): 317-323.

[9] Chechile RA. 2003. Mathematical tools for hazard function analysis. Journal of Mathematical Psychology, 47(5-6): 478-494.

[10] Cheng RCH \& AMin NAK. 1979. Maximum product-of-spacings estimation with applications to the lognormal distribution. Tech. Rep. 1, University of Wales IST, Department of Mathematics.

[11] Cheng RCH \& AMIN NAK. 1983. Estimating parameters in continuous univariate distributions with a shifted origin. Journal of the Royal Statistical Society, Series B45(3): 394-403.

[12] David HA \& Moeschberger ML. 1978. The Theory of Competing Risks. Vol. 39 of Griffin's Statistical Monograph Series. Macmillan Co., New York.

[13] Doornik JA. 2007. Object-Oriented Matrix Programming Using Ox, 3rd ed. London: Timberlake Consultants Press and Oxford.

[14] Ghitany M, Al-Mutairi D, Balakrishnan N \& Al-Enezi L. 2013. Power lindley distribution and associated inference. Computational Statistics \& Data Analysis, 64: 20-33.

[15] Ghitany ME, Al-Mutairi DK, Al-Awadhi FA \& Al-Burais MM. 2012. Marshall-Olkin extended Lindley distribution and its application. International Journal of Applied Mathematics, 25(5): 709-721.

[16] Ghitany ME, Al-Mutairi DK \& NADARAJah S. 2008. Zero-truncated Poisson-Lindley distribution and its application. Mathematics and Computers in Simulation, 79(3): 279-287.

[17] Ghitany ME \& Al-Mutari DK. 2008. Size-biased Poisson-Lindley distribution and its application. METRON - International Journal of Statistics, 66(3): 299-311.

[18] Ghitany ME, Alqallaf F, Al-Mutairi DK \& Husain HA. 2011. A two-parameter weighted Lindley distribution and its applications to survival data. Mathematics and Computers in Simulation, 81: 1190-1201. 
[19] Ghitany ME, Atieh B \& Nadarajah S. 2008. Lindley distribution and its application. Mathematics and Computers in Simulation, 78(4): 493-506.

[20] GLASER RE. 1980. Bathtub and related failure rate characterizations. Journal of the American Statistical Association, 75(371): 667-672.

[21] Hemmati F, Khorram E \& ReZakhah S. 2011. A new three-parameter ageing distribution. Journal of Statistical Planning and Inference, 141(7): 2266-2275.

[22] JodRÁ P. 2010. Computer generation of random variables with Lindley or Poisson-Lindley distribution via the Lambert W function. Mathematics and Computers in Simulation, 81(4): 851-859.

[23] KUş C. 2007. A new lifetime distribution. Computational Statistics \& Data Analysis, 51(9): 44974509 .

[24] LeE ET \& WANG JW. 2003. Statistical methods for survival data analysis, 3rd Edition. Wiley Series in Probability and Statistics. Hoboken, NJ.

[25] Lindley D. 1965. Introduction to Probability and Statistics from a Bayesian Viewpoint, Part II: Inference. Cambridge University Press, New York.

[26] LindLeY DV. 1958. Fiducial distributions and Bayes' theorem. It Journal of the Royal Statistical Society. Series B. Methodological, 20: 102-107.

[27] LU W \& SHI D. 2012. A new compounding life distribution: the Weibull-Poisson distribution. Journal of Applied Statistics, 39(1): 21-38.

[28] LUCEÑO A. 2006. Fitting the generalized pareto distribution to data using maximum goodness-of-fit estimators. Computational Statistics \& Data Analysis, 51(2): 904-917.

[29] Mahmoudi E \& Zakerzadeh H. 2010. Generalized Poisson Lindley distribution. Communications in Statistics Theory and Methods, 39: 1785-1798.

[30] MaZuCheli J \& ACHCAR JA. 2011. The Lindley distribution applied to competing risks lifetime data. Computer Methods and Programs in Biomedicine, 104(2): 188-192.

[31] NadARAJAh S, BAKouch HS \& TAhmasbi R. 2011. A generalized Lindley distribution. Sankhyãa, B 73(2): 331-359.

[32] Oluyede B \& YAng T. 2015. A new class of generalized lindley distributions with applications. Journal of Statistical Computation and Simulation, 85(10): 2072-2100.

[33] RANNEB Y B. 1984. The maximum spacing method. An estimation method related to the maximum likelihood method. Scandinavian Journal of Statistics. Theory and Applications, 11(2): 93-112.

[34] Rezaei S \& TAhmasbi R. 2012. A new lifetime distribution with increasing failure rate: Exponential truncated Poisson. Journal of Basic and Applied Scientific Research, 2(2): 1749-1762.

[35] Ristić MM \& NADARAJAH S. 2012. A new lifetime distribution. Journal of Statistical Computation and Simulation iFirst, 1-16.

[36] Sankaran M. 1970. The discrete Poisson-Lindley distribution. Biometrics, 26: 145-149.

[37] SAS. 2011. SAS/ETS ${ }^{\circledR}$ User's Guide, Version 9.33. Cary, NC: SAS Institute Inc.

[38] Zakerzadeh H \& Dolati A. 2009. Generalized Lindley distribution. Journal of Mathematical Extension, 3: 13-25.

[39] Zamani H \& Ismail N. 2010. Negative Binomial-Lindley distribution and its application. Journal of Mathematics and Statistics, 6(1): 4-9. 